\title{
Article \\ Cyano- and Ketone-Containing Selenoesters as Multi-Target Compounds against Resistant Cancers
}

\author{
Nikoletta Szemerédi ${ }^{1}{ }^{\circledR}$, Simona Dobiasová ${ }^{2}$, Noemi Salardón-Jiménez ${ }^{3} \mathbb{D}$, Annamária Kincses ${ }^{1}{ }^{\circledR}$, \\ Márta Nové ${ }^{1}$, Giyaullah Habibullah ${ }^{2}{ }^{\oplus}$, Clotilde Sevilla-Hernández ${ }^{3}{ }^{\oplus}$, Miguel Benito-Lama $^{3}$, \\ Francisco-Javier Alonso-Martínez ${ }^{3}$, Jitka Viktorová ${ }^{2, *} *$, Gabriella Spengler ${ }^{1, *}(\mathbb{B})$ \\ and Enrique Domínguez-Álvarez ${ }^{3, * \mathbb{B}}$
}

Citation: Szemerédi, N.; Dobiasová, S.; Salardón-Jiménez, N.; Kincses, A.; Nové, M.; Habibullah, G.; Sevilla-Hernández, C.; Benito-Lama, M.; Alonso-Martínez, F.-J.; Viktorová, J.; et al. Cyano- and Ketone-Containing Selenoesters as Multi-Target Compounds against Resistant Cancers Cancers 2021, 13, 4563. https:// doi.org/10.3390/cancers13184563

Academic Editor: Fortunato Ciardiello

Received: 12 July 2021

Accepted: 8 September 2021

Published: 11 September 2021

Publisher's Note: MDPI stays neutral with regard to jurisdictional claims in published maps and institutional affiliations.

Copyright: (c) 2021 by the authors. Licensee MDPI, Basel, Switzerland. This article is an open access article distributed under the terms and conditions of the Creative Commons Attribution (CC BY) license (https:// creativecommons.org/licenses/by/ $4.0 /)$.
1 Department of Medical Microbiology, Albert Szent-Györgyi Health Center and Faculty of Medicine, University of Szeged, Semmelweis utca 6, 6725 Szeged, Hungary; szemeredi.nikoletta@med.u-szeged.hu (N.S.); kincses.annamaria90@gmail.com (A.K.); nove.marta@med.u-szeged.hu (M.N.)

2 Department of Biochemistry and Microbiology, Faculty of Food and Biochemical Technology, University of Chemistry and Technology Prague, Technická 3, 16628 Prague 6, Czech Republic; dobiasoo@vscht.cz (S.D.); habibuli@vscht.cz (G.H.)

3 Instituto de Química Orgánica General (IQOG-CSIC), Consejo Superior de Investigaciones Científicas, Juan de la Cierva 3, 28006 Madrid, Spain; noemi.sj.95@gmail.com (N.S.-J.); clo.sh.1995@gmail.com (C.S.-H.); miguelbenitodelama@gmail.com (M.B.-L.); franalonso9112@gmail.com (F.-J.A.-M.)

* Correspondence: prokesoj@vscht.cz (J.V.); spengler.gabriella@med.u-szeged.hu (G.S.); e.dominguez-alvarez@iqog.csic.es (E.D.-Á.)

Simple Summary: The search for novel anticancer agents has been the hot topic of interest in cancer research, due to the phenomenon of multidrug resistance (MDR) in cancer that can make cancer cells resistant to the current available chemotherapeutic agents. In this context, we have designed, synthesized, and biologically evaluated 15 novel selenoesters, with the aim to explore their activity against resistant cancer cell lines. Some of these described selenocompounds showed noteworthy cytotoxicity and selectivity, the ability to inhibit the ABCB1 efflux pump, the capacity to modulate the ATPase activity of this pump, the capability to trigger apoptotic events, the ability to interact in a synergistic manner with doxorubicin in resistant cancer cells, and the power to promote wound healing. Consequently, these results validate the design of these selenocompounds and justify further research to evaluate the possibilities of these compounds to be used in the future in the fight against resistant cancers.

Abstract: Fifteen selenocompounds, comprising of eight ketone-containing selenoesters (K1-K8, also known as oxoselenoesters) and seven cyano-containing selenoesters (N1-N7, known also as cyanoselenoesters), have been designed, synthesized, and evaluated as novel anticancer agents. These compounds are derivatives of previously reported active selenoesters and were prepared following a three-step one-pot synthetic route. The following evaluations were performed in their biological assessment: cytotoxicity determination, selectivity towards cancer cells in respect to noncancer cells, checkerboard combination assay, ABCB1 inhibition and inhibition of ABCB1 ATPase activity, apoptosis induction, and wound healing assay. As key results, all the compounds showed cytotoxicity against cancer cells at low micromolar concentrations, with cyanoselenoesters being strongly selective. All of the oxoselenoesters, except K4, were potent ABCB1 inhibitors, and two of them, namely $\mathbf{K} \mathbf{5}$ and $\mathbf{K} 6$, enhanced the activity of doxorubicin in a synergistic manner. The majority of these ketone derivatives modulated the ATPase activity, showed wound healing activity, and induced apoptosis, with $\mathrm{K} 3$ being the most potent, with a potency close to that of the reference compound. To summarize, these novel derivatives have promising multi-target activity, and are worthy to be studied more in-depth in future works to gain a greater understanding of their potential applications against cancer. 
Keywords: multidrug resistance; efflux pump; ABCB1; apoptosis; selenium; cancer

\section{Introduction}

The occurrence of multidrug resistance (MDR) to chemotherapeutic drugs has become a significant challenge in cancer therapy. One of the most important factors contributing to MDR is the overexpression of efflux pumps. P-glycoprotein (P-gp)—also known as multidrug resistance protein 1 (MDR1), ATP-binding cassette sub-family B member 1 (ABCB1), or the cluster of differentiation 243 (CD243) - was discovered in 1970 as a member of the ATP-binding cassette (ABC) transporter family [1,2]. These ABC transporters fulfil physiological functions in the gastrointestinal tract, liver, and lungs. They are localized in different barriers that separate blood vessels from specific organs, such as the blood-brain barrier (BBB), the blood-cerebrospinal fluid (B-CSF), the blood-retina barrier (BRB), the blood-testis barrier (BTB), and in the placenta [3]. In humans, this transporter is encoded by the MDR1, also known as $A B C B 1$ gene [4]. ABCB1 substrates are typically amphiphilic compounds. ABCB1 is comprised of two nucleotide-binding domains and 12 transmembrane domains which constitute a drug-binding pocket. This transporter is a natural cell protective protein whose function is the removal of xenobiotic compound out of the cells, as this compound can be toxic for the cells $[5,6]$. ABCB1 can remove various chemotherapeutic agents, e.g., daunorubicin, doxorubicin, vinblastine, vincristine, epirubicin, etoposide, imatinib, irinotecan, paclitaxel, or colchicine, thus leading to treatment failure in anticancer therapy [7]. It has been reported that $\mathrm{ABCB} 1$, together with the multidrug resistance-associated protein 1 (MRP1)/ATP Binding Cassette Subfamily C Member 1 ( $\mathrm{ABCC} 1$ ) (encoded by $A B C C 1$ gene), is the major determinant of innate drug sensitivity, even at the lowest level of expression $[8,9]$. The design of inhibitors of efflux pumps, especially in regard to ABCB1, is a promising strategy in cancer therapy [10].

Selenium, and the organic and inorganic compounds that contain this element, are essential in various biological processes. It is known that selenium deficiency can cause disorders or augment risk of developing cancers [11]. Alternatively, epidemiological studies reported that dietary supplementation with selenium can reduce the incidence of certain types of cancers. These starting works in selenium supplementation led to the reporting of a wide variety of organic and inorganic selenocompounds with chemopreventive, antiproliferative, and cytotoxic activity against cancer [12]. Sodium selenite is probably the most deeply studied inorganic selenium salt with anticancer activity. Among organic selenocompounds with chemopreventive and anticancer activity, methylselenol, methylseleninic acid, selenocyanates, and diphenyl diselenide can be cited [13]. Finally, the number of works that study the anticancer and multidrug resistance reversing activities of selenium nanoparticles is significantly increasing nowadays [14].

Considering these lines of evidence, we have designed, synthesized, and determined the biological activity of selenium-containing anticancer agents, mostly of them selenoesters. It has been demonstrated that a selenoanhydride derivative and some selenoester derivatives have potent anticancer activity against $\mathrm{ABCB} 1$ expressing in MDR mouse T-lymphoma cells and MDR colon adenocarcinoma cells due to ABCB1 inhibition and apoptosis induction [15]. In addition, these derivatives exerted potent anticancer activity on sensitive and resistant breast cancer cell lines [16]. It has been confirmed that selenium compounds synergistically enhance the activity of anticancer drugs when they are administered in combination [17]. Furthermore, as a drug-repurposing approach, phenothiazines with a known pharmacological and toxicity profile were combined with the previously mentioned selenoanhydride and selenoester, and several Se-compounds exhibited synergistic activity in combination with promethazine, chlorpromazine, and thioridazine [18]. The synergistic effects observed suggest that selenium compounds are able to reverse MDR and potentiate the activity of reference anticancer drugs or compounds with well-defined anticancer activity. 
Based on these results, 15 newly synthesized selenoesters, shown in Figure 1, have been investigated in this study with regard to their anticancer and MDR reversing capacity in sensitive and resistant colon adenocarcinoma cell lines. Out of them, eight contain a ketone in the alkyl group directly bound to the selenium atom (oxoselenoesters or ketone-selenoesters, K1-K8); and seven contain a cyano group in the same alkyl moiety (cyanoselenoesters, N1-N7).

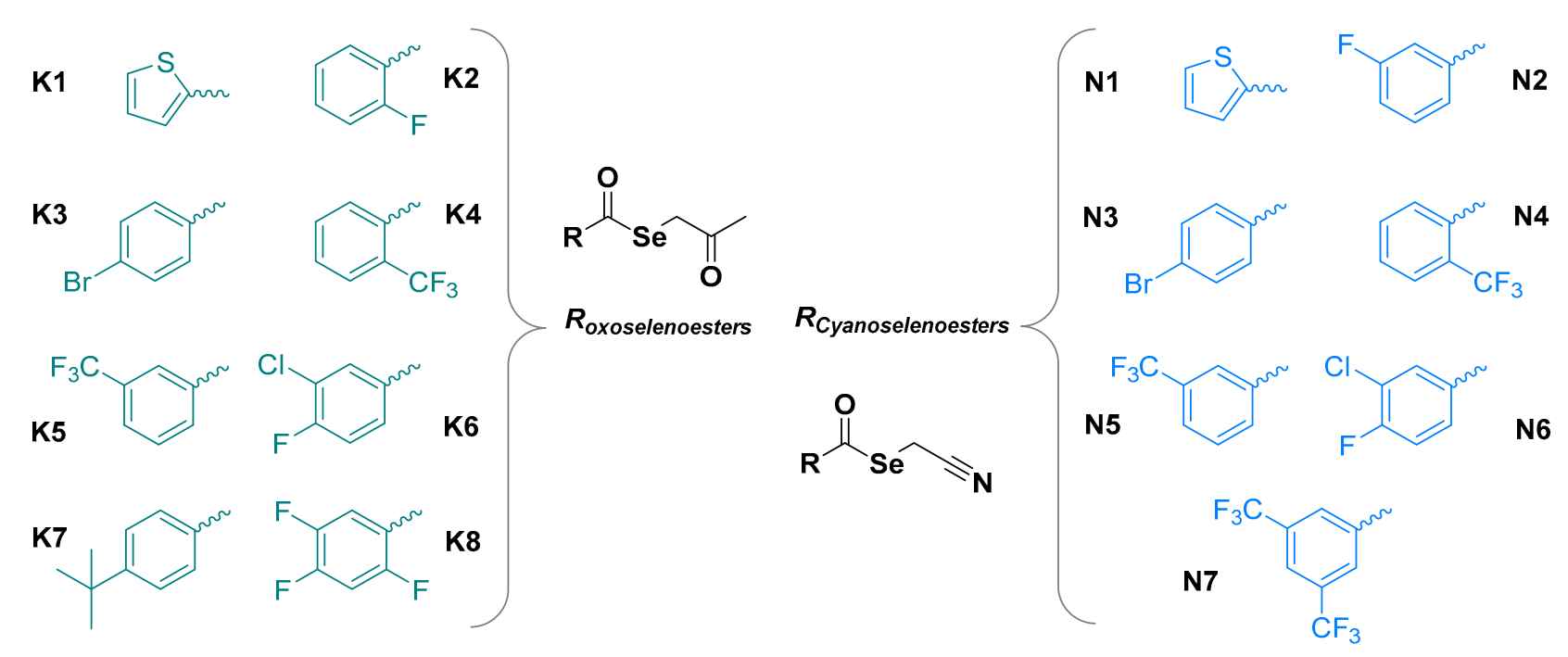

Figure 1. Structure of the oxoselenoesters, K1-K8, and of the cyanoselenoesters, N1-N7, presented in this work.

\section{Materials and Methods}

\subsection{Chemical Reagents and Chemical Characterization}

The chemical reagents, solvents, and materials used to synthesize the cyanoselenoesters and the oxoselenoesters presented herein were acquired at different vendors: Acros Organics and Alfa Aesar (both brands of Thermo Fisher Scientific, Geel, Belgium); Fluorochem (Hadfield, Derbyshire, UK); Honeywell Riedel de Haën (Seelze, Germany); Panreac Química S.L.U (Castellar del Vallés, Barcelona, Spain); Scharlab S.L. Spain (Sentmenat, Barcelona, Spain); and Sigma-Aldrich Merck S.L.U. Spain (Madrid, Spain).

To characterize the compounds, preliminary NMR spectra (Nuclear Magnetic Resonance) were taken using a Varian Inova-300 spectrometer (Agilent Technologies, Santa Clara, CA, USA) to monitor the reactions. The purity of the compounds, with a clean spectrum at the $300 \mathrm{MHz}$ spectrometer, was assessed by means of elemental analysis using a LECO CHNS-932 microanalyser (LECO Europe B.V., Geleen, The Netherlands), at a temperature of $990{ }^{\circ} \mathrm{C}$, using $\mathrm{He}$ as a carrier gas, and using silver capsules to introduce the sample in the analyser. Each compound must show a deviation of less than $0.40 \%$ in each element analysed $(\mathrm{C}, \mathrm{H}, \mathrm{N}, \mathrm{S})$ to be considered pure. The spectra included in the Supplementary Materials (Figures S1A-S15F) were taken with the following instruments: (i) NMR- ${ }^{1} \mathrm{H}$, NMR $-{ }^{13} \mathrm{C}$, and bidimensional COSY (Correlation spectroscopy); HSQC (Heteronuclear Single-Quantum Correlation spectroscopy); HMBC (Heteronuclear Multiple-Bond Correlation spectroscopy); and a Bruker Avance III HD-400 (Billerica, MA, USA) spectrometer using TMS as the internal standard. Next, (ii) mass spectra with a direct insertion probe (MS-DIP); and a quadrupole HP 5973 MSD spectrometer (Hewlett Packard, now Agilent Technologies, Santa Clara, CA, USA) with a direct insertion probe and electronic impact (EI) in positive mode as the ionization source, at a $70 \mathrm{eV}$ ionization energy and with an $m / z$ precision of \pm 0.05 . Finally, (iii) Infrared spectroscopy (IR), using a Spectrum One B (Perkin-Elmer, Waltham, MA, USA) spectrophotometer. Solid samples were assayed preparing $\mathrm{KBr}$ films, whereas liquid samples were assayed between $\mathrm{NaCl}$ crystals. Finally, melting points have been determined in a Reichert-Kofler heating system coupled with a microscope; they are provided as obtained by visual inspection, without correction. 


\subsection{Synthetic Procedure}

A synthetic procedure with three consecutive reactions in the same pot has been followed to obtain these 2-oxopropyl selenoesters and cyanomethyl selenoesters. In a first step, an equivalent of selenium grey powder is suspended in $20 \mathrm{~mL}$ of water, and 2 equivalents of sodium borohydride are added slowly over it and left stirring, usually for $15 \mathrm{~min}$, or until the end of the gas release. Subsequently, an equivalent of the corresponding acyl chloride is added, and the reaction is stirred for $90 \mathrm{~min}$ at $50-70{ }^{\circ} \mathrm{C}$. Then, the crude reaction mixture is filtered to eliminate the formed boron salts, and an equivalent of the appropriate alkyl halide are added at that time over the filtrate. The mixture is then kept reacting until reaction completion, which normally requires $1 \mathrm{~h}$ at $50{ }^{\circ} \mathrm{C}$ and an additional hour, initially at room temperature, and later ice-cooled.

The desired selenoester is isolated and purified by application of the most adequate techniques in each case, namely, as precipitation if the compound is solid, and extraction plus column chromatography if it is liquid. The purification followed for each compound is described in Section 2.3.

Starting acyl chlorides, if not commercially available at a reasonable cost, can be synthesized through the chlorination of the corresponding carboxylic acid with thionyl chloride. In this case, the appropriate derivative of benzoic acid is solved in an excess of thionyl chloride (at least 5:1 in molar ratio) and kept refluxing while stirring for $5 \mathrm{~h}$. Then, thionyl chloride is removed in the rotary evaporator and the crude is washed 3 times with $50 \mathrm{~mL}$ of toluene, removing the toluene each time in the rotary evaporator to take away the remaining amounts of thionyl chloride. The structure of the synthesized acyl chloride is verified by ${ }^{1} \mathrm{H}-\mathrm{NMR}$, as compared with published data (data not shown). Then, the reagent is used in the synthesis of the respective selenocompound without further purification.

\subsection{Chemical Description of the Compounds}

\subsubsection{Se-(2-Oxopropyl) Thiophene-2-carboselenoate (K1)}

The reagents used: sodium borohydride $(0.119 \mathrm{~g}, 3.15 \mathrm{mmol})$, grey selenium $(0.120 \mathrm{~g}$, $1.52 \mathrm{mmol})$, thiophene-2-carbonyl chloride $(0.219 \mathrm{~g}, 0.16 \mathrm{~mL}, 1.5 \mathrm{mmol})$, and chloroacetone $(0.139 \mathrm{~g}, 0.12 \mathrm{~mL}, 1.5 \mathrm{mmol})$. The final compound precipitated as a yellow solid powder that was isolated by filtration and washed with water, rendering $181 \mathrm{mg}(49 \%)$. MW: 247.17. Mp: $52-53^{\circ} \mathrm{C}$. DIP-MS $m / z$ (abundance \%): 57.05 (3); 83.05 (7); 111.05 (100); and 245.95/2.47.95 (1/2, Se, $\left.\mathrm{M}^{+}\right)$. IR (KBr) (cm $\left.{ }^{-1}\right)$ : $3082\left(\mathrm{~m}, \mathrm{C}-\mathrm{H}_{\mathrm{Ar}}\right) ; 2958,2922(\mathrm{~m}, \mathrm{C}-\mathrm{H}) ; 1704$ (m, C=O ketone); 1661 (s, C=O selenoester); and 1645, 1514 (m, C-C $\left.\mathrm{Ar}_{\mathrm{Ar}}\right){ }^{1} \mathbf{H}-\mathrm{NMR}(400 \mathrm{MHz}$, $\left.\mathrm{CDCl}_{3}\right) ; \delta: 7.83\left(\mathrm{dd}, \mathrm{J}_{3-4}=3.9 \mathrm{~Hz}, \mathrm{~J}_{3-5}=1.0 \mathrm{~Hz}, 1 \mathrm{H}, \mathrm{H}_{3}\right) ; 7.72\left(\mathrm{dd}, J_{4-5}=5.0 \mathrm{~Hz}, 1 \mathrm{H}, \mathrm{H}_{5}\right) ; 7.16$ $\left(\mathrm{dd}, 1 \mathrm{H}, \mathrm{H}_{4}\right) ; 3.90\left(\mathrm{~s}, 2 \mathrm{H}, \mathrm{SeCH}_{2}\right)$; and $2.33\left(\mathrm{~s}, 3 \mathrm{H}, \mathrm{COCH}_{3}\right) .{ }^{13} \mathrm{C}-\mathrm{NMR}\left(101 \mathrm{MHz}, \mathrm{CDCl}_{3}, \delta\right.$ : $203.6\left(\mathrm{COCH}_{3}\right) ; 183.0(\mathrm{COSe}) ; 142.7\left(\mathrm{C}_{2}\right) ; 134.2\left(\mathrm{C}_{5}\right) ; 132.5\left(\mathrm{C}_{3}\right) ; 128.3\left(\mathrm{C}_{4}\right) ; 34.5\left(\mathrm{SeCH}_{2}\right)$; and $28.7\left(\mathrm{COCH}_{3}\right)$. Elemental analysis for $\mathrm{C}_{8} \mathrm{H}_{8} \mathrm{O}_{2} \mathrm{SSe}$, calculated/found (\%): $\mathrm{C}: 38.88 / 39.18$; H: 3.26/3.41; and S:12.97/12.93.

\subsubsection{Se-(2-Oxopropyl) 2-Fluorobenzoselenoate (K2)}

The reagents used: sodium borohydride $(0.122 \mathrm{~g}, 3.22 \mathrm{mmol})$, grey selenium $(0.123 \mathrm{~g}$, $1.55 \mathrm{mmol})$, 2-fluorobenzoyl chloride $(0.239 \mathrm{~g}, 0.18 \mathrm{~mL}, 1.51 \mathrm{mmol})$, and chloroacetone $(0.139 \mathrm{~g}, 0.12 \mathrm{~mL}, 1.50 \mathrm{mmol})$. The final compound was obtained as a pale-yellow liquid, rendering $59 \mathrm{mg}(\mathbf{1 5 \%})$. MW: 259.14. Mp: Liquid RT. DIP-MS $m / z$ (abundance \%): 75.05 (16); 95.05 (38); 123.15 (100); 161.05 (8); and 255.95/256.95/257.95/259.95/261.95 $\left(0 / 0 / 1 / 2 / 0, \mathrm{Se}, \mathrm{M}^{+}\right)$. IR (KBr) $\left(\mathrm{cm}^{-1}\right)$ : 3067, $3005\left(\mathrm{~s}, \mathrm{C}-\mathrm{H}_{\mathrm{Ar}}\right) ; 2925(\mathrm{~m}, \mathrm{C}-\mathrm{H}) ; 1707(\mathrm{~m}$, $\mathrm{C}=\mathrm{O}$ ketone); 1656 (s, C=O selenoester); and 1609, 1577, 1482, 1452 (m, C- $\left.\mathrm{C}_{\mathrm{Ar}}\right) .{ }^{1} \mathrm{H}-\mathrm{NMR}$ $\left(400 \mathrm{MHz}, \mathrm{CDCl}_{3}\right), \delta: 7.86\left(\mathrm{td}, J_{6-5}=7.6 \mathrm{~Hz}, J_{6-4}=1.8 \mathrm{~Hz}, 1 \mathrm{H}, \mathrm{H}_{6}\right) ; 7.58\left(\mathrm{dddd}, J_{4-3}=8.3 \mathrm{~Hz}\right.$, $\left.J_{4-5}=7.5 \mathrm{~Hz}, J_{4-F}=5.0 \mathrm{~Hz}, J_{4-6}=1.7 \mathrm{~Hz}, 1 \mathrm{H}, \mathrm{H}_{4}\right) ; 7.26\left(\mathrm{td}, 1 \mathrm{H}, \mathrm{H}_{5}\right), 7.19\left(\mathrm{ddd}, J_{3-F}=11.0 \mathrm{~Hz}\right.$, $\left.1 \mathrm{H}, \mathrm{H}_{3}\right) ; 3.90\left(\mathrm{~s}, 2 \mathrm{H}, \mathrm{SeCH}_{2}\right)$; and $2.35\left(\mathrm{~s}, 3 \mathrm{H}, \mathrm{COCH}_{3}\right) .{ }^{13} \mathrm{C}-\mathrm{NMR}\left(101 \mathrm{MHz}, \mathrm{CDCl}_{3}, \delta\right.$ : $203.7\left(\mathrm{COCH}_{3}\right) ; 188.4\left(\mathrm{~d}, J_{\text {COSe }-F}=5.2 \mathrm{~Hz}, \mathrm{COSe}\right) ; 160.9\left(\mathrm{~d}, J_{C(F)-F}=258.7 \mathrm{~Hz}, \mathrm{C}_{2(\mathrm{~F})}\right) ; 135.4$ $\left(\mathrm{d}, J_{6-F}=9.2 \mathrm{~Hz}, \mathrm{C}_{6}\right) ; 129.5\left(\mathrm{~d}, J_{5-F}=1.4 \mathrm{~Hz}, \mathrm{C}_{5}\right) ; 126.0\left(\mathrm{~d}, J_{1-F}=10.4 \mathrm{~Hz}, \mathrm{C}_{1}\right) ; 124.8(\mathrm{~d}$, $\left.J_{4-F}=3.6 \mathrm{~Hz}, \mathrm{C}_{4}\right) ; 117.2\left(\mathrm{~d}, J_{3-\mathrm{F}}=22.2 \mathrm{~Hz}, \mathrm{C}_{3}\right) ; 34.8\left(\mathrm{~d}, J_{\mathrm{SeCH} 2-\mathrm{F}}=6.5 \mathrm{~Hz}, \mathrm{SeCH}_{2}\right)$; and 28.9 
$\left(\mathrm{COCH}_{3}\right)$. Elemental analysis for $\mathrm{C}_{10} \mathrm{H}_{9} \mathrm{FO}_{2} \mathrm{Se}$, calculated/found (\%): C: 46.35/46,54; and H: 3.50/3.55.

\subsubsection{Se-(2-Oxopropyl) 4-Bromobenzoselenoate (K3)}

The reagents used: sodium borohydride $(0.200 \mathrm{~g}, 5.29 \mathrm{mmol})$, grey selenium $(0.200 \mathrm{~g}$, $2.53 \mathrm{mmol})$, 4-bromobenzoyl chloride $(0.551 \mathrm{~g}, 2.51 \mathrm{mmol})$, and chloroacetone $(0.230 \mathrm{~g}$, $0.20 \mathrm{~mL} 2.51 \mathrm{mmol})$. The final compound precipitated as a white-pale-pink solid that was isolated by filtration, washed with water, and dried, rendering $331 \mathrm{mg}$ (41\%). MW: 320.04. Mp: $55-57{ }^{\circ} \mathrm{C}$. DIP-MS $m / z$ (abundance \%): 50.15 (10); 75.05 (16); 76.05 (18); 154.95/156.95 (31/30, Br); 182.95/184.95 (100/97, Br); and 317.90/319.90/31.85 (0/1/1, $\left.\mathrm{Br}+\mathrm{Se}, \mathrm{M}^{+}-2 / \mathrm{M}^{+} / \mathrm{M}^{+\cdot}+2\right)$. IR $(\mathrm{KBr})\left(\mathrm{cm}^{-1}\right): 2954,2917\left(\mathrm{w}, \mathrm{C}-\mathrm{H}_{\mathrm{Alk}}\right) ; 1706(\mathrm{~m}, \mathrm{C}=\mathrm{O}$ ketone); 1665 (s, C=O selenoester); and 1582, 1564, 1481 (m, C-C $\left.\mathrm{Cr}_{\mathrm{Ar}}\right) .{ }^{\mathbf{1}} \mathrm{H}-\mathrm{NMR}(400 \mathrm{MHz}$, $\left.\mathrm{CDCl}_{3}\right) ; \delta: 7.76\left(\mathrm{td}, J_{2-3,6-5}=8.7 \mathrm{~Hz}, J_{2-B r, 6-B r}=2.3 \mathrm{~Hz}, 2 \mathrm{H}, \mathrm{H}_{2}+\mathrm{H}_{6}\right) ; 7.62\left(\mathrm{td}, J_{3-B r, 5-B r}=2.3 \mathrm{~Hz}\right.$, $\left.2 \mathrm{H}, \mathrm{H}_{3}+\mathrm{H}_{5}\right) ; 3.91\left(\mathrm{~s}, 2 \mathrm{H}, \mathrm{SeCH}_{2}\right)$; and $2.34\left(\mathrm{~s}, 3 \mathrm{H}, \mathrm{COCH}_{3}\right) .{ }^{13} \mathrm{C}-\mathrm{NMR}\left(101 \mathrm{MHz} \mathrm{CDCl}_{3}\right.$,

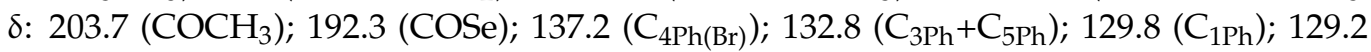
$\left(\mathrm{C}_{2 \mathrm{Ph}}+\mathrm{C}_{6 \mathrm{Ph}}\right)$; $35.1\left(\mathrm{SeCH}_{2}\right)$; and $29.2\left(\mathrm{COCH}_{3}\right)$. Elemental analysis for $\mathrm{C}_{10} \mathrm{H}_{9} \mathrm{BrO}_{2} \mathrm{Se}$, calculated/found (\%): C: 37.53/37.53; and H: 2.83/2.78.

\subsubsection{Se-(2-Oxopropyl) 2-(Trifluoromethyl)benzoselenoate (K4)}

The reagents used: sodium borohydride $(0.197 \mathrm{~g}, 5.21 \mathrm{mmol})$, grey selenium $(0.195 \mathrm{~g}$, $2.47 \mathrm{mmol}), 2$-(trifluoromethyl)benzoyl chloride $(0.523 \mathrm{~g}, 0.37 \mathrm{~mL}, 2.51 \mathrm{mmol})$, and chloroacetone $(0.239 \mathrm{~g}, 0.20 \mathrm{~mL}, 2.5 \mathrm{mmol})$. The final compound was extracted with dichloromethane $(4 \times 30 \mathrm{~mL})$, dried, and evaporated in vacuum. A column using dichloromethane-toluene (3:1) was performed to purify the compound, rendering $158 \mathrm{mg}$ of a pale-yellow liquid (21\%). MW: 247.17. Mp: Liquid RT. DIP-MS $m / z$ (abundance \%): 75.05 (5); 95.05 (8); 125.05 (6); 126.05 (3); 145.05 (59); 173.15 (100); and 307.95/309.95 (0/0, Se, M M $^{+\cdot}$. IR (KBr) $\left(\mathrm{cm}^{-1}\right)$ : 3074, $3006\left(\mathrm{~m}, \mathrm{C}-\mathrm{H}_{\mathrm{Ar}}\right) ; 2925\left(\mathrm{~m}, \mathrm{C}-\mathrm{H}_{\mathrm{Alk}}\right) ; 1697$ (s, broad, overlapping bands of selenoester and ketone $\mathrm{C}=\mathrm{O})$; and 1601, 1582, 1448 (w, C-C $\mathrm{Ar}) .{ }^{\mathbf{1}} \mathbf{H}-\mathbf{N M R}\left(400 \mathrm{MHz}, \mathrm{CDCl}_{3}\right)$;

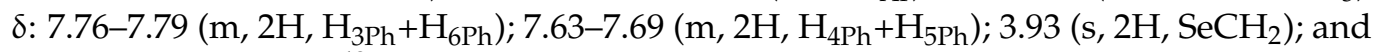
$2.35\left(\mathrm{~s}, 3 \mathrm{H}, \mathrm{COCH}_{3}\right) .{ }^{13} \mathrm{C}-\mathrm{NMR}\left(101 \mathrm{MHz}_{2} \mathrm{CDCl}_{3}, \delta: 203.3\left(\mathrm{COCH}_{3}\right) ; 193.3\right.$ (COSe); 138.6 $\left(\mathrm{q}, J_{1-C F 3}=1.8 \mathrm{~Hz}, \mathrm{C}_{1 \mathrm{Ph}}\right) ; 132.1\left(\mathrm{~d}, J_{4-C F 3}=0.7 \mathrm{~Hz}, \mathrm{C}_{4 \mathrm{Ph}}\right) ; 132.0\left(\mathrm{C}_{5 \mathrm{Ph}}\right), 128.4\left(\mathrm{C}_{6 \mathrm{Ph}}\right) ; 127.4$ $\left(\mathrm{q}, J_{3-C F 3}=5.3 \mathrm{~Hz}, \mathrm{C}_{3 \mathrm{Ph}}\right) ; 126.4\left(\mathrm{q}, J_{6-C F 3}=33.0 \mathrm{~Hz}, \mathrm{C}_{2 \mathrm{Ph}}\right) ; 121.8\left(\mathrm{q}, J_{C-F[C F 3]}=274.5 \mathrm{~Hz}, \mathrm{CF}_{3}\right)$; $36.0\left(\mathrm{SeCH}_{2}\right)$; and $28.6\left(\mathrm{COCH}_{3}\right)$. Elemental analysis for $\mathrm{C}_{9} \mathrm{H}_{11} \mathrm{~F}_{3} \mathrm{O}_{2} \mathrm{Se}$, calculated/found (\%): C: 42.74/42.42; and H: 2.93/3.09.

\subsubsection{Se-(2-Oxopropyl) 3-(Trifluoromethyl)benzoselenoate (K5)}

The reagents used: sodium borohydride $(0.119 \mathrm{~g}, 3.15 \mathrm{mmol})$, grey selenium $(0.120 \mathrm{~g}$, $1.52 \mathrm{mmol}), 3$-(trifluoromethyl)benzoyl chloride $(0.313 \mathrm{~g}, 0.23 \mathrm{~mL}, 1.53 \mathrm{mmol})$, and chloroacetone $(0.139 \mathrm{~g}, 0.12 \mathrm{~mL}, 1.50 \mathrm{mmol})$. The final compound was extracted with dichloromethane $(4 \times 30 \mathrm{~mL})$, dried, and evaporated in vacuum. A column using dichloromethane-toluene (3:1) was performed to purify the compound, rendering $133 \mathrm{mg}$ of a pale-yellow liquid (29\%). MW: 238.15. Mp: Liquid RT. DIP-MS m/z (abundance \%): 75.05 (6); 95.05 (9); 125.05 (7); 126.05 (5); 145.05 (74); 173.15 (100); and 305.95/306.95/307.95/309.95/311.95 $\left(0 / 0 / 1 / 2 / 0\right.$, Se, $\left.\mathrm{M}^{+}\right)$. IR $(\mathrm{KBr})\left(\mathrm{cm}^{-1}\right): 3073,3007\left(\mathrm{~m}, \mathrm{C}-\mathrm{H}_{\mathrm{Ar}}\right) ; 2925\left(\mathrm{~m}, \mathrm{C}-\mathrm{H}_{\mathrm{Alk}}\right) ; 1712$ (s, C=O ketone); 1679 (s, C=O selenoester); and 1611, 1590, 1484 (m, C-C ${ }_{\text {Ar }}$ ). ${ }^{1}$ H-NMR $\left(400 \mathrm{MHz}_{2} \mathrm{CDCl}_{3}\right) ; \delta: 8.14\left(\mathrm{~s}, 1 \mathrm{H}, \mathrm{H}_{2 \mathrm{Ph}}\right) ; 8.08\left(\mathrm{~d}, J_{6-5}=7.9 \mathrm{~Hz}, 1 \mathrm{H}, \mathrm{H}_{6 \mathrm{Ph}}\right) ; 7.88\left(\mathrm{~d}, J_{4-5}=7.8 \mathrm{~Hz}\right.$, $\left.1 \mathrm{H}, \mathrm{H}_{4 \mathrm{Ph}}\right) ; 7.64\left(\mathrm{t}, 1 \mathrm{H}, \mathrm{H}_{5 \mathrm{Ph}}\right) ; 3.96\left(\mathrm{~s}, 2 \mathrm{H}, \mathrm{SeCH}_{2}\right)$; and $2.36\left(\mathrm{~s}, 3 \mathrm{H}, \mathrm{COCH}_{3}\right) .{ }^{13} \mathrm{C}-\mathrm{NMR}$ $\left(101 \mathrm{MHz}, \mathrm{CDCl}_{3}, \delta: 203.1\left(\mathrm{COCH}_{3}\right) ; 192.1(\mathrm{COSe}) ; 138.8\left(\mathrm{C}_{1 \mathrm{Ph}}\right) ; 131.9\left(\mathrm{q}, J_{3-\mathrm{CF} 3}=33.3 \mathrm{~Hz}\right.\right.$, $\left.\mathrm{C}_{3 \mathrm{Ph}}\right) ; 130.7\left(\mathrm{~d}, J_{6-C F 3}=1.0 \mathrm{~Hz}, \mathrm{C}_{6 \mathrm{Ph}}\right) ; 130.6\left(\mathrm{q}, \mathrm{J}_{4-\mathrm{CF} 3}=3.5 \mathrm{~Hz}, \mathrm{C}_{4 \mathrm{Ph}}\right) ; 129.9\left(\mathrm{C}_{5 \mathrm{Ph}}\right) ; 124.2$ $\left(\mathrm{q}, J_{2-C F 3}=3.9 \mathrm{~Hz}, \mathrm{C}_{2 \mathrm{Ph}}\right) ; 123.5\left(\mathrm{q}, J_{C-F[C F 3]}=272.5 \mathrm{~Hz}, \mathrm{CF}_{3}\right) ; 35.0\left(\mathrm{SeCH}_{2}\right) ;$ and 28.9 $\left(\mathrm{COCH}_{3}\right)$. Elemental analysis for $\mathrm{C}_{9} \mathrm{H}_{6} \mathrm{ClNOSe}$, calculated/found (\%): C: 42.74/42.43; and H: 2.93/3.05. The acyl chloride (3-fluorobenzoyl chloride) was synthesized from 3fluorobenzoic acid $(15.859 \mathrm{~g}, 113.19 \mathrm{mmol})$ and thionyl chloride (50 mL, excess), obtaining 
$11.675 \mathrm{~g}$ of the acyl chloride (65\% yield). Complete yield of the full synthetic route is then $19 \%$.

\subsubsection{Se-(2-Oxopropyl) 3-Chloro-4-fluorobenzoselenoate (K6)}

The reagents used: sodium borohydride $(0.159 \mathrm{~g}, 4.20 \mathrm{mmol})$, grey selenium $(0.161 \mathrm{~g}$, $2.04 \mathrm{mmol})$, 3-chloro-4-fluorobenzoyl chloride $(0.389 \mathrm{~g}, 2.02 \mathrm{mmol})$, and chloroacetone $(0.186 \mathrm{~g}, 0.16 \mathrm{~mL}, 2.01 \mathrm{mmol})$. The final compound precipitated as a white solid that was isolated by filtration, washed with water, and purified in the column chromatography using dichloromethane as the eluent; obtaining, after column, $106 \mathrm{mg}$ of a white solid powder (18\%). MW: 238.15. Mp: $42-44^{\circ} \mathrm{C}$. DIP-MS $m / z$ (abundance \%): 74.05 (3); 93.05 (5); 94.05 (7); $109.05 / 110.95$ (5/2, Cl); $129.05 / 130.95$ (9/5, Cl); 157.05/158.95 (100/33, Cl); and 289.95/290.95/291.95/293.95/295.95 (0/0/1/1/0, Se, $\left.\mathrm{M}^{+*}\right)$. IR $(\mathrm{KBr})\left(\mathrm{cm}^{-1}\right): 3097$, 3050 (m, C-H $\mathrm{Hr}_{\mathrm{Ar}}$ ); 2978, 2898 (m, C-H); 1717 (m, C=O ketone); 1672 (s, C=O selenoester); and 1591, $1494\left(\mathrm{~m}, \mathrm{C}-\mathrm{C}_{\mathrm{Ar}}\right) .{ }^{1} \mathrm{H}-\mathrm{NMR}\left(400 \mathrm{MHz}, \mathrm{CDCl}_{3}\right) ; \delta: 7.97\left(\mathrm{dd}, J_{2-F}=6.9 \mathrm{~Hz}, J_{2-6}=2.2 \mathrm{~Hz}\right.$, $\left.1 \mathrm{H}, \mathrm{H}_{2 \mathrm{Ph}}\right) ; 7.82\left(\mathrm{ddd}, J_{6-5}=8.6 \mathrm{~Hz}, J_{6-F}=4.4 \mathrm{~Hz}, 1 \mathrm{H}, \mathrm{H}_{6 \mathrm{Ph}}\right) ; 7.25\left(\mathrm{t}, 1 \mathrm{H}, \mathrm{H}_{5 \mathrm{Ph}}\right) ; 3.93(\mathrm{~s}$, 2H, $\left.\mathrm{SeCH}_{2}\right)$; and $2.34\left(\mathrm{~s}, 3 \mathrm{H}, \mathrm{COCH}_{3}\right) .{ }^{13} \mathrm{C}-\mathrm{NMR}\left(101 \mathrm{MHz}, \mathrm{CDCl}_{3}, \delta: 203.1\left(\mathrm{COCH}_{3}\right)\right.$; 190.6 (COSe); $161.7\left(\mathrm{~d}, J_{C 4(F)-F}=258.7 \mathrm{~Hz}, \mathrm{C}_{4 \mathrm{Ph}(\mathrm{F})}\right) ; 135.3\left(\mathrm{~d}, J_{1-F}=3.7 \mathrm{~Hz}, \mathrm{C}_{1 \mathrm{Ph}}\right) ; 130.1$ $\left(\mathrm{d}, J_{2-F}=1.1 \mathrm{~Hz}, \mathrm{C}_{2 \mathrm{Ph}}\right) ; 127.9\left(\mathrm{~d}, J_{6-F}=8.6 \mathrm{~Hz}, \mathrm{C}_{6 \mathrm{Ph}}\right) ; 122.6\left(\mathrm{~d}, J_{3-F}=18.5 \mathrm{~Hz}, \mathrm{C}_{3 \mathrm{Ph}(\mathrm{Cl})}\right)$; $117.4\left(\mathrm{~d}, J_{5-F}=22.1 \mathrm{~Hz}, \mathrm{C}_{5 \mathrm{Ph}}\right) ; 35.1\left(\mathrm{SeCH}_{2}\right)$; and $28.9\left(\mathrm{COCH}_{3}\right)$. Elemental analysis for $\mathrm{C}_{10} \mathrm{H}_{8} \mathrm{ClFO}_{2} \mathrm{Se}$, calculated/found (\%): C: 40.91/40.65; and $\mathrm{H}: 2.75 / 2.83$. The acyl chloride (3-chloro-4-fluorobenzoyl chloride) was synthesized from 3-chloro-4-fluorobenzoic acid (9.426 $\mathrm{g}, 50 \mathrm{mmol})$ and thionyl chloride (35 mL, excess), obtaining $9.602 \mathrm{~g}$ of the acyl chloride (99.5\% yield). Complete yield of the full synthetic route is then $18 \%$.

\subsubsection{Se-(2-Oxopropyl) 4-(Tert-butyl)benzoselenoate (K7)}

The reagents used: sodium borohydride $(0.119 \mathrm{~g}, 3.15 \mathrm{mmol})$, grey selenium $(0.119 \mathrm{~g}$, $1.51 \mathrm{mmol})$, 4-tert-butylbenzoyl chloride $(0.292 \mathrm{~g}, 1.47 \mathrm{mmol})$, and chloroacetone $(0.139 \mathrm{~g}$, $0.12 \mathrm{~mL}, 1.50 \mathrm{mmol})$. The final compound was extracted with dichloromethane $(4 \times 30 \mathrm{~mL})$, dried, and evaporated in vacuum. A column using dichloromethane-hexane (4:1) was performed to purify the compound, rendering $30 \mathrm{mg}$ of a pale-yellow liquid (7\%). MW: 297.26. Mp: Liquid RT. DIP-MS m/z (abundance \%): 65.05 (1); 77.05 (4); 91.05 (9); 105.05 (5); 118.05 (15); 133.15 (2); 146.05 (17); 161.15 (100); and 298.05 (0, Se, M M $^{+\cdot}$. IR (KBr) $\left(\mathrm{cm}^{-1}\right): 2964,2907,2870$ (s, C-H); 1710 (s, C=O ketone); 1678 (s, C=O selenoester); and 1601, 1567, 1465 (m, C-C Ar $) .{ }^{1} \mathrm{H}-\mathrm{NMR}\left(400 \mathrm{MHz}, \mathrm{CDCl}_{3}\right) ; \delta: 7.85\left(\mathrm{~d}, J_{2-3,6-5}=8.6 \mathrm{~Hz}, 2 \mathrm{H}\right.$, $\left.\mathrm{H}_{2}+\mathrm{H}_{6}\right) ; 7.49\left(\mathrm{~d}, 2 \mathrm{H}, \mathrm{H}_{3}+\mathrm{H}_{5}\right) ; 3.89\left(\mathrm{~s}, 2 \mathrm{H}, \mathrm{SeCH}_{2}\right) ; 2.33\left(\mathrm{~s}, 3 \mathrm{H}, \mathrm{COCH}_{3}\right) ;$ and $1.34(\mathrm{~s}, 9 \mathrm{H}$, $\left.\mathrm{C}_{4} \mathrm{H}_{9}\right) .{ }^{13} \mathrm{C}-\mathrm{NMR}\left(101 \mathrm{MHz}, \mathrm{CDCl}_{3}, \delta: 204.1\left(\mathrm{COCH}_{3}\right) ; 192.2(\mathrm{COSe}) ; 158.4\left(\mathrm{C}_{1}\right) ; 135.5\left(\mathrm{C}_{4}\right)\right.$; $127.5\left(\mathrm{C}_{2}+\mathrm{C}_{6}\right) ; 126.1\left(\mathrm{C}_{3}+\mathrm{C}_{5}\right) ; 35.5\left(\underline{\mathrm{C}}\left(\mathrm{CH}_{3}\right)_{3}\right) ; 34.2\left(\mathrm{SeCH}_{2}\right) ; 31.2\left(\mathrm{C}\left(\mathrm{CH}_{3}\right)_{3}\right) ;$ and $28.6(\mathrm{~s}$, $\left.\mathrm{COCH}_{3}\right)$. Elemental analysis for $\mathrm{C}_{14} \mathrm{H}_{18} \mathrm{O}_{2} \mathrm{Se}$, calculated/found (\%): C: 56.57/56.52; and H: 6.10/5.97.

\subsubsection{Se-(2-Oxopropyl) 2,4,5-Trifluorobenzoselenoate (K8)}

The reagents used: sodium borohydride $(0.199 \mathrm{~g}, 5.26 \mathrm{mmol})$, grey selenium $(0.200 \mathrm{~g}$, $2.53 \mathrm{mmol}), 2,4,5$-trifluorobenzoyl chloride $(0.483 \mathrm{~g}, 2.48 \mathrm{mmol})$, and chloroacetone $(0.244 \mathrm{~g}$, $0.21 \mathrm{~mL}, 2.63 \mathrm{mmol})$. The final compound precipitated as a yellow solid powder that was isolated by filtration and washed with water, rendering $306 \mathrm{mg}$ (42\%). MW: 295.12. Mp: 40-43 ${ }^{\circ}$ C. DIP-MS $m / z$ (abundance \%): 81.05 (10); 92.95(1); 112.05 (1); 131.05 (20); 159.05 (100); and 291.95/292.95/293.95/295.95/297.95 (0/0/1/1/0, Se, M+. IR (KBr) $\left(\mathrm{cm}^{-1}\right)$ : 3058 (s, C-H $\mathrm{Hr}_{\mathrm{Ar}}$ ); 2963, 2921 (m, C-H); 1708 (s, C=O ketone); 1650 (s, C=O selenoester); and 1623, 1511 (s, C-C $\mathrm{Ar}_{\text {) }}$. ${ }^{1} \mathrm{H}-\mathrm{NMR}\left(400 \mathrm{MHz}, \mathrm{CDCl}_{3}\right) ; \delta: 7.71\left(\mathrm{ddd}, J_{6-F 5}=10.1 \mathrm{~Hz}, J_{6-F 4}=8.6 \mathrm{~Hz}\right.$, $\left.\mathrm{J}_{6-\mathrm{F} 2}=6.4,1 \mathrm{H}, \mathrm{H}_{6}\right) ; 7.07\left(\mathrm{td}, J_{3-F 4,3-F 2}=9.8 \mathrm{~Hz}, J_{3-F 5}=6.1 \mathrm{~Hz}, 1 \mathrm{H}, \mathrm{H}_{3}\right) ; 3.92\left(\mathrm{~s}, 2 \mathrm{H}, \mathrm{SeCH}_{2}\right)$; and $2.35\left(\mathrm{~s}, 3 \mathrm{H}, \mathrm{COCH}_{3}\right) .{ }^{13} \mathrm{C}-\mathrm{NMR}\left(101 \mathrm{MHz} \mathrm{CDCl}_{3}, \delta: 203.1\right.$ (s, $\left.\mathrm{COCH}_{3}\right) ; 186.5$ (s, COSe); $157.2\left(\mathrm{ddd}, J_{2-F 2}=257.6 \mathrm{~Hz}, J_{2-F 4}=10.1 \mathrm{~Hz}, J_{2-F 5}=2.5 \mathrm{~Hz}, \mathrm{C}_{2(\mathrm{~F})}\right) ; 153.6\left(\mathrm{ddd}, J_{4-F 4}=261.5 \mathrm{~Hz}\right.$, $\left.J_{4-F 5}=14.7 \mathrm{~Hz}, J_{4-F 2}=12.5 \mathrm{~Hz}, \mathrm{C}_{4(\mathrm{~F})}\right) ; 147.1\left(\mathrm{ddd}, J_{5-F 5}=249.5 \mathrm{~Hz}, J_{5-F 4}=12.9 \mathrm{~Hz}, J_{5-F 2}=3.3 \mathrm{~Hz}\right.$, $\left.\mathrm{C}_{2(\mathrm{~F})}\right) ; 122.4\left(\mathrm{ddd}, J_{1-F 2}=13.1 \mathrm{~Hz}, J_{1-F 5}=4.3 \mathrm{~Hz}, J_{1-F 4}=3.8 \mathrm{~Hz}, \mathrm{C}_{1(\mathrm{~F})}\right) ; 117.3\left(\mathrm{dt}, J_{6-F 5}=20.5 \mathrm{~Hz}\right.$, 
$\left.J_{6}-F 2,6-F 4=3.1 \mathrm{~Hz}, \mathrm{C}_{6}\right) ; 107.3\left(\mathrm{dd}, J_{3-F 4}=28.6 \mathrm{~Hz}, J_{3-F 6}=21.3 \mathrm{~Hz}, \mathrm{C}_{3}\right) ; 35.1\left(\mathrm{SeCH}_{2}\right) ;$ and 29.1 $\left(\mathrm{COCH}_{3}\right)$. Elemental analysis for $\mathrm{C}_{10} \mathrm{H}_{7} \mathrm{~F}_{3} \mathrm{O}_{2} \mathrm{Se}$, calculated / found (\%): $\mathrm{C}: 40.70 / 40.61$; and $\mathrm{H}: 2.39 / 2.45$. The acyl chloride (2,4,5-trifluorobenzoyl chloride) was synthesized from 2,4,5trifluorobenzoic acid $(8.805 \mathrm{~g}, 50 \mathrm{mmol})$ and thionyl chloride ( $35 \mathrm{~mL}$, excess), obtaining $9.538 \mathrm{~g}$ of the acyl chloride ( $98 \%$ yield). Complete yield of the full synthetic route is then $41 \%$.

\subsubsection{Se-(Cyanomethyl) Thiophene-2-carboselenoate (N1)}

The reagents used: sodium borohydride $(0.197 \mathrm{~g}, 5.21 \mathrm{mmol})$, grey selenium $(0.194 \mathrm{~g}$, $2.46 \mathrm{mmol})$, thienyl chloride $(0.370 \mathrm{~g}, 0.27 \mathrm{~mL}, 2.54 \mathrm{mmol})$, and chloroacetonitrile $(0.191 \mathrm{~g}$, $0.16 \mathrm{~mL}, 2.53 \mathrm{mmol})$. The final compound precipitated as a beige solid that was isolated by filtration and washed with water, rendering $85 \mathrm{mg}$ (15\% yield). MW: $230.14 . \mathrm{Mp}$ : $50-52{ }^{\circ} \mathrm{C}$. DIP-MS $m / z$ (abundance \%): 57.05 (9); 83.05 (21); 111.15 (100); and $230.95\left(0, \mathrm{M}^{+}\right)$. IR (KBr, $\left.\mathrm{cm}^{-1}\right)$ : 3111, 3082, $3068\left(\mathrm{w}, \mathrm{C}-\mathrm{H}_{\mathrm{Ar}}\right) ; 2990,2935\left(\mathrm{~m}, \mathrm{C}-\mathrm{H}_{\mathrm{Alk}}\right) ; 2246(\mathrm{~m}, \mathrm{C} \equiv \mathrm{N}) ; 1662(\mathrm{~s}, \mathrm{C}=\mathrm{O})$; and $1512,1406\left(\mathrm{~m}, \mathrm{C}-\mathrm{C}_{\text {Thiophene }}\right){ }^{1} \mathbf{H}-\mathbf{N M R}\left(400 \mathrm{MHz}, \mathrm{CDCl}_{3}\right) ; \delta: 7.81\left(\mathrm{dd}, J_{2-3}=3.9 \mathrm{~Hz}\right.$, $\left.J_{2-4}=1.1 \mathrm{~Hz}, 1 \mathrm{H}, \mathrm{H}_{2 \mathrm{Tp}}\right) ; 7.81\left(\mathrm{dd}, J_{2-4}=5.0 \mathrm{~Hz}, 1 \mathrm{H}, \mathrm{H}_{4 \mathrm{Tp}}\right) ; 7.19\left(\mathrm{dd}, 1 \mathrm{H}, \mathrm{H}_{3 \mathrm{Tp}}\right) ;$ and $3.71(\mathrm{~s}$, 2H, $\left.\mathrm{SeCH}_{2}\right) .{ }^{13} \mathrm{C}-\mathrm{NMR}\left(101 \mathrm{MHz}, \mathrm{CDCl}_{3}, \delta: 180.4(\mathrm{CO}) ; 141.5\left(\mathrm{C}_{1 \mathrm{Tp}}\right) ; 135.2\left(\mathrm{C}_{4 \mathrm{Tp}}\right) ; 133.0\right.$ $\left(\mathrm{C}_{2 \mathrm{Tp}}\right) ; 128.5\left(\mathrm{C}_{3 \mathrm{Tp}}\right) ; 117.2(\mathrm{C} \equiv \mathrm{N}) ;$ and $5.5\left(\mathrm{SeCH}_{2}\right)$. Elemental analysis for $\mathrm{C}_{7} \mathrm{H}_{5} \mathrm{NOSSe}$, calculated/found (\%): C: 36.53/36.69; H: 2.19/2.32; N: 6.09/6.22; and S: 13.93/13.89.

\subsubsection{Se-(Cyanomethyl) 3-Fluorobenzoselenoate (N2)}

The reagents used: sodium borohydride $(0.199 \mathrm{~g}, 5.26 \mathrm{mmol})$, grey selenium $(0.198 \mathrm{~g}$, $2.51 \mathrm{mmol})$, 3-fluorobenzoyl chloride $(0.391 \mathrm{~g}, 0.30 \mathrm{~mL}, 2.47 \mathrm{mmol})$, and chloroacetonitrile $(0.190 \mathrm{~g}, 0.16 \mathrm{~mL}, 2.53 \mathrm{mmol})$. The final compound was extracted with dichloromethane $(4 \times 30 \mathrm{~mL})$, dried, and evaporated in vacuum. A column using dichloromethane-hexane (4:1) was performed to purify the compound, rendering $138 \mathrm{mg}(23 \%)$ of a pale-yellow liquid. MW: 242.11. Mp: Liquid at room temperature. DIP-MS $m / z$ (abundance \%): 50.15 (6); 69.05 (6); 75.15 (35); 95.15 (93); $123.15(100)$; and $242.95\left(0, \mathrm{M}^{+}\right) . \mathbf{I R}\left(\mathrm{NaCl}, \mathrm{cm}^{-1}\right)$ : $3075(\mathrm{w}$, C- $\left.\mathrm{H}_{\mathrm{Ar}}\right) ; 2998,2944\left(\mathrm{~m}, \mathrm{C}-\mathrm{H}_{\mathrm{Alk}}\right) ; 2246(\mathrm{~m}, \mathrm{C} \equiv \mathrm{N}) ; 1690(\mathrm{~s}, \mathrm{C}=\mathrm{O})$; and 1589, 1482, $1437(\mathrm{~m}, \mathrm{C}-$ $\left.\mathrm{C}_{\mathrm{Ar}}\right) \cdot{ }^{1} \mathrm{H}-\mathrm{NMR}\left(400 \mathrm{MHz}, \mathrm{CDCl}_{3}\right) ; \delta: 7.66\left(\mathrm{td}, J_{6-5}=7.8 \mathrm{~Hz}, J_{6-2}=1.0 \mathrm{~Hz}, 1 \mathrm{H}, \mathrm{H}_{6 \mathrm{Ph}}\right) ; 7.55(\mathrm{dt}$, $\left.J_{2-F}=9.1 \mathrm{~Hz}, J_{2-4}=2.3 \mathrm{~Hz}, 1 \mathrm{H}, \mathrm{H}_{2 \mathrm{Ph}}\right) ; 7.50\left(\mathrm{td}, J_{5-4,5-6}=8.2 \mathrm{~Hz}, J_{5-F}=5.6 \mathrm{~Hz}, 1 \mathrm{H}, \mathrm{H}_{5 \mathrm{Ph}}\right) ; 7.37$ (tdd, $\left.1 \mathrm{H}, \mathrm{H}_{4 \mathrm{Ph}}\right)$; and $3.71\left(\mathrm{~s}, 2 \mathrm{H}, \mathrm{SeCH}_{2}\right) .{ }^{13} \mathrm{C}-\mathrm{NMR}\left(101 \mathrm{MHz}, \mathrm{CDCl}_{3}, \delta: 189.5\right.$ (CO); 163.0 $\left(\mathrm{d}, J_{3-F}=250.4 \mathrm{~Hz}, \mathrm{C}_{3 \mathrm{Ph}}\right) ; 139.1\left(\mathrm{~d}, J_{2-F}=6.6 \mathrm{~Hz}, \mathrm{C}_{1 \mathrm{Ph}}\right) ; 131.1\left(\mathrm{~d}, J_{5-F}=7.8 \mathrm{~Hz}, \mathrm{C}_{5 \mathrm{Ph}}\right) ; 123.5$ $\left(\mathrm{d}, J_{6-F}=3.1 \mathrm{~Hz}, \mathrm{C}_{6 \mathrm{Ph}}\right) ; 121.9\left(\mathrm{~d}, J_{4-\mathrm{F}}=21.5 \mathrm{~Hz}, \mathrm{C}_{4 \mathrm{Ph}}\right) ; 117.1(\mathrm{C} \equiv \mathrm{N}), 114.2\left(\mathrm{~d}, J_{2-F}=23.3 \mathrm{~Hz}\right.$, $\left.\mathrm{C}_{2 \mathrm{Ph}}\right)$; and $5.7\left(\mathrm{SeCH}_{2}\right)$. Elemental analysis for $\mathrm{C}_{9} \mathrm{H}_{6} \mathrm{FNOSe}$, calculated/found (\%): $\mathrm{C}$ : 44.65/44.42; H: 2.50/2.62; and N: 5.79/6.83. The acyl chloride (3-fluorobenzoyl chloride) was synthesized from 3-fluorobenzoic acid $(15.859 \mathrm{~g}, 113.19 \mathrm{mmol})$ and thionyl chloride ( $50 \mathrm{~mL}, 82 \mathrm{~g}$, excess), obtaining $11.675 \mathrm{~g}$ of the acyl chloride (65\% yield). Complete yield of the full synthetic route is then $15 \%$.

\subsubsection{Se-(Cyanomethyl) 4-Bromobenzoselenoate (N3)}

The reagents used: sodium borohydride $(0.200 \mathrm{~g}, 5.29 \mathrm{mmol})$, grey selenium $(0.199 \mathrm{~g}$, $2.52 \mathrm{mmol})$, 4-bromobenzoyl chloride $(0.551 \mathrm{~g}, 2.51 \mathrm{mmol})$, and chloroacetonitrile $(0.191 \mathrm{~g}$, $0.16 \mathrm{~mL}, 2.53 \mathrm{mmol})$. The final compound precipitated as a white solid that was isolated by filtration and washed with water, rendering $340 \mathrm{mg}(45 \%)$. MW: $303.02 . \mathrm{Mp}: 105-107^{\circ} \mathrm{C}$. DIP-MS $m / z$ (abundance \%): 50.10 (14); 75.00 (23); 76.00 (23); 154.90/156.90 (37/37, Br); and 182.90/184.90 (100/98, Br). IR $\left(\mathrm{KBr}, \mathrm{cm}^{-1}\right): 3082,3007\left(\mathrm{~m}, \mathrm{C}-\mathrm{H}_{\mathrm{Ar}}\right) ; 2241(\mathrm{~m}, \mathrm{C} \equiv \mathrm{N})$; 1667 (s, C=O); and 1586, 1565, $1394\left(\mathrm{~m}, \mathrm{C}-\mathrm{C}_{\mathrm{Ar}}\right) .{ }^{1} \mathrm{H}-\mathrm{NMR}\left(400 \mathrm{MHz}, \mathrm{CDCl}_{3}\right) ; \delta: 7.73(\mathrm{td}$, $\left.J_{2-3,6-5}=8.7 \mathrm{~Hz}, J_{2-B r, 6-B r}=2.0 \mathrm{~Hz}, 2 \mathrm{H}, \mathrm{H}_{2}+\mathrm{H}_{6}\right) ; 7.66\left(\mathrm{td}, J_{3-B r, 5-B r}=2.1 \mathrm{~Hz}, 2 \mathrm{H}, \mathrm{H}_{3}+\mathrm{H}_{5}\right)$; and $3.70\left(\mathrm{~s}, 2 \mathrm{H}, \mathrm{SeCH}_{2}\right) .{ }^{13} \mathrm{C}-\mathrm{NMR}\left(101 \mathrm{MHz}, \mathrm{CDCl}_{3}, 8: 189.6\right.$ (COSe); $136.0\left(\mathrm{C}_{4 \mathrm{Ph}(\mathrm{Br})}\right) ; 131.7$ $\left(\mathrm{C}_{3 \mathrm{Ph}}+\mathrm{C}_{5 \mathrm{Ph}}\right) ; 130.2\left(\mathrm{C}_{1 \mathrm{Ph}}\right) ; 128.9\left(\mathrm{C}_{2 \mathrm{Ph}}+\mathrm{C}_{6 \mathrm{Ph}}\right) ; 117.1(\mathrm{C} \equiv \mathrm{N})$; and $5.6\left(\mathrm{SeCH}_{2}\right)$. Elemental analysis for $\mathrm{C}_{9} \mathrm{H}_{6}$ BrNOSe, calculated/found (\%): C: $35.67 / 35.37$; $\mathrm{H}: 2.00 / 2.03$; and N: 4.62/4.72. 


\subsubsection{Se-(Cyanomethyl) 2-(Trifluoromethyl)benzoselenoate (N4)}

The reagents used: $\mathrm{m}$ sodium borohydride $(0.197 \mathrm{~g}, 5.21 \mathrm{mmol})$, grey selenium $(0.197 \mathrm{~g}$, $2.49 \mathrm{mmol})$, 2-(trifluoromethyl)benzoyl chloride $(0.524 \mathrm{~g}, 2.51 \mathrm{mmol})$, and chloroacetonitrile $(0.191 \mathrm{~g}, 0.16 \mathrm{~mL}, 2.53 \mathrm{mmol})$. The final compound precipitated as a white powder that was isolated by filtration and washed with water, rendering $294 \mathrm{mg}$ (40\%). MW: 292.12 . Mp: 65-67 ${ }^{\circ} \mathrm{C}$. DIP-MS $m / z$ (abundance \%): 50.15 (4); 75.05 (11); 95.05 (15); 125.05 (11); 126.05 (5); 145.05 (88); and 173.15 (100). IR (KBr, cm $\left.{ }^{-1}\right)$ : 2992, 2936 (w, C-H $\left.\mathrm{H}_{\mathrm{Alk}}\right) ; 2239$ (s, $\mathrm{C} \equiv \mathrm{N}) ; 1706(\mathrm{~s}, \mathrm{C}=\mathrm{O})$; and 1584, $1390\left(\mathrm{~m}, \mathrm{C}-\mathrm{C}_{\mathrm{Ar}}\right) .{ }^{1} \mathrm{H}-\mathrm{NMR}\left(400 \mathrm{MHz}, \mathrm{CDCl}_{3}\right) ; \delta: 7.81(\mathrm{dd}$, $\left.J_{6-5}=5.4 \mathrm{~Hz}, J_{6-4}=3.7 \mathrm{~Hz}, 1 \mathrm{H}, \mathrm{H}_{6 \mathrm{Ph}}\right) ; 7.76\left(\mathrm{dd}, J_{3-4}=5.5 \mathrm{~Hz}, J_{3-5}=3.8 \mathrm{~Hz}, 1 \mathrm{H}, \mathrm{H}_{3 \mathrm{Ph}}\right) ; 7.71$ $\left(\mathrm{t}, 1 \mathrm{H}, \mathrm{H}_{4 \mathrm{Ph}}\right) ; 7.69\left(\mathrm{t}, 1 \mathrm{H}, \mathrm{H}_{5 \mathrm{Ph}}\right)$; and $3.72\left(\mathrm{~s}, 2 \mathrm{H}, \mathrm{SeCH}_{2}\right) .{ }^{13} \mathrm{C}-\mathrm{NMR}\left(101 \mathrm{MHz}, \mathrm{CDCl}_{3}, \delta\right.$ : $191.2(\mathrm{COSe}) ; 137.3\left(\mathrm{C}_{1 \mathrm{Ph}}\right) ; 132.6\left(\mathrm{C}_{5 \mathrm{Ph}}\right) ; 132.4\left(\mathrm{~d}, J_{4-\mathrm{CF} 3}=0.7 \mathrm{~Hz}, \mathrm{C}_{4 \mathrm{Ph}}\right) ; 128.4\left(\mathrm{C}_{6 \mathrm{Ph}}\right) ; 127.6$ $\left(\mathrm{q}, J_{5-C F 3}=5.3 \mathrm{~Hz}, \mathrm{C}_{3 \mathrm{Ph}}\right) ; 126.8\left(\mathrm{q}, J_{6-\mathrm{CF} 3}=33.1 \mathrm{~Hz}, \mathrm{C}_{2 \mathrm{Ph}}\right) ; 123.0\left(\mathrm{q}, J_{\mathrm{C}-\mathrm{F}[C F 3]}=274.1 \mathrm{~Hz}, \mathrm{CF}_{3}\right)$; $116.8(\mathrm{C} \equiv \mathrm{N})$; and $7.0\left(\mathrm{SeCH}_{2}\right)$. Elemental analysis for $\mathrm{C}_{10} \mathrm{H}_{6} \mathrm{~F}_{3} \mathrm{NOSe}$, calculated / found (\%): C: 41.12/41.08; H: 2.07/2.15; and N: 4.79/4.75.

\subsubsection{Se-(Cyanomethyl) 3-(Trifluoromethyl)benzoselenoate (N5)}

The reagents used: sodium borohydride $(0.198 \mathrm{~g}, 5.23 \mathrm{mmol})$, grey selenium $(0.198 \mathrm{~g}$, $2.51 \mathrm{mmol})$, 3-(trifluoromethyl)benzoyl chloride ( $0.526 \mathrm{~g}, 2.52 \mathrm{mmol})$, and chloroacetonitrile $(0.191 \mathrm{~g}, 0.16 \mathrm{~mL}, 2.53 \mathrm{mmol})$. The final compound was a liquid non-miscible with water that was separated by decantation. Crude liquid was dissolved in $100 \mathrm{~mL}$ of dichloromethane and treated with silica, activated charcoal, and anhydrous sodium sulfate. After filtration and removal of the solvent in a rotary, $157 \mathrm{mg}(\mathbf{2 1 \%})$ of a pale-yellow liquid was obtained. MW: 292.12. Mp: Liquid at room temperature. DIP-MS $m / z$ (abundance \%): 50.15 (5); 75.05 (10); 95.05 (13); 125.05 (9); 126.05 (5); 145.05 (85); 173.15 (100); and 292.95 $\left(0, \mathrm{M}^{+\cdot}\right)$. IR $\left(\mathrm{KBr}, \mathrm{cm}^{-1}\right)$ : 3074, $3000\left(\mathrm{~s}, \mathrm{C}-\mathrm{H}_{\mathrm{Ar}}\right) ; 2946\left(\mathrm{~m}, \mathrm{C}-\mathrm{H}_{\mathrm{Alk}}\right) ; 2247(\mathrm{~s}, \mathrm{C} \equiv \mathrm{N}) ; 1683$ (s, C=O); and 1612, 1440 (s, C-C $\mathrm{Ar}) .{ }^{1} \mathrm{H}-\mathrm{NMR}\left(400 \mathrm{MHz}, \mathrm{CDCl}_{3}\right) ; \delta: 8.11\left(\mathrm{~s}, 1 \mathrm{H}, \mathrm{H}_{2 \mathrm{Ph}}\right)$; $8.05\left(\mathrm{~d}, J_{6-5}=7.9 \mathrm{~Hz}, 1 \mathrm{H}, \mathrm{H}_{6 \mathrm{Ph}}\right) ; 7.93\left(\mathrm{~d}, J_{4-5}=7.8 \mathrm{~Hz}, 1 \mathrm{H}, \mathrm{H}_{4 \mathrm{Ph}}\right) ; 7.68\left(\mathrm{t}, 1 \mathrm{H}, \mathrm{H}_{5 \mathrm{Ph}}\right)$; and $3.74\left(\mathrm{~s}, 2 \mathrm{H}, \mathrm{SeCH}_{2}\right) .{ }^{13} \mathrm{C}-\mathrm{NMR}\left(101 \mathrm{MHz}, \mathrm{CDCl}_{3}, \delta: 189.7(\mathrm{COSe}) ; 137.9\left(\mathrm{C}_{1 \mathrm{Ph}}\right) ; 132.2(\mathrm{q}\right.$, $\left.J_{3-C F 3}=33.5 \mathrm{~Hz}, \mathrm{C}_{3 \mathrm{Ph}}\right) ; 131.2\left(\mathrm{q}, J_{4-\mathrm{CF} 3}=3.5 \mathrm{~Hz}, \mathrm{C}_{4 \mathrm{Ph}}\right) ; 130.8\left(\mathrm{~d}, J_{6-\mathrm{CF} 3}=1.0 \mathrm{~Hz}, \mathrm{C}_{6 \mathrm{Ph}}\right) ; 130.2$ $\left(\mathrm{C}_{5 \mathrm{Ph}}\right) ; 124.3\left(\mathrm{q}, J_{2-C F 3}=3.8 \mathrm{~Hz}, \mathrm{C}_{2 \mathrm{Ph}}\right) ; 123.4\left(\mathrm{q}, J_{\mathrm{C}-F[C F 3]}=272.8 \mathrm{~Hz}, \mathrm{CF}_{3}\right) ; 116.9(\mathrm{CN})$; and $5.9\left(\mathrm{SeCH}_{2}\right)$. Elemental analysis for $\mathrm{C}_{10} \mathrm{H}_{6} \mathrm{~F}_{3} \mathrm{NOSe}$, calculated/found (\%): $\mathrm{C}: 41.12 / 41.13$; H: 2.07/2.12; and N: 4.79/4.83.

\subsubsection{Se-(Cyanomethyl) 3-Chloro-4-fluorobenzoselenoate (N6)}

The reagents used: sodium borohydride $(0.200 \mathrm{~g}, 5.29 \mathrm{mmol})$, grey selenium $(0.197 \mathrm{~g}$, $2.49 \mathrm{mmol}), 3$-chloro-4-fluorobenzoyl chloride $(0.484 \mathrm{~g}, 2.51 \mathrm{mmol})$, and chloroacetonitrile $(0.191 \mathrm{~g}, 0.16 \mathrm{~mL}, 2.53 \mathrm{mmol})$. The final compound precipitated as a white powder that was isolated by filtration and washed with water, rendering $279 \mathrm{mg}(\mathbf{4 1} \%)$. MW: 276.55 . Mp: $73-75^{\circ}$ C. DIP-MS $m / z$ (abundance \%): 74.00 (4); 93.00 (8); 94.00 (9); 109.00/110.95 (7/2, Cl); 129.00/130.95 (38/12, Cl); and 156.90/158.90 (100/33, Cl). IR (KBr, cm $\left.{ }^{-1}\right)$ : 3101, 3051, $3010\left(\mathrm{~s}, \mathrm{C}-\mathrm{H}_{\mathrm{Ar}}\right) ; 2953\left(\mathrm{~m}, \mathrm{C}-\mathrm{H}_{\mathrm{Alk}}\right) ; 2242(\mathrm{~s}, \mathrm{C} \equiv \mathrm{N}) ; 1679(\mathrm{~s}, \mathrm{C}=\mathrm{O}) ;$ and 1587, 1495, 1398 $\left(\mathrm{s}, \mathrm{C}-\mathrm{C}_{\mathrm{Ar}}\right) .{ }^{1} \mathrm{H}-\mathrm{NMR}\left(400 \mathrm{MHz}, \mathrm{CDCl}_{3}\right) ; \delta: 7.94\left(\mathrm{dd}, J_{2-F}=6.8 \mathrm{~Hz}, J_{2-6}=2.2 \mathrm{~Hz}, 1 \mathrm{H}, \mathrm{H}_{2 \mathrm{Ph}}\right)$; $7.79\left(\mathrm{ddd}, \mathrm{J}_{6-5}=8.5 \mathrm{~Hz}, \mathrm{~J}_{6-\mathrm{F}}=4.3 \mathrm{~Hz}, 1 \mathrm{H}, \mathrm{H}_{6 \mathrm{Ph}}\right) ; 7.29\left(\mathrm{t}, 1 \mathrm{H}, \mathrm{H}_{5 \mathrm{Ph}}\right)$; and $3.71\left(\mathrm{~s}, 2 \mathrm{H}, \mathrm{SeCH}_{2}\right)$. ${ }^{13} \mathrm{C}-\mathrm{NMR}\left(101 \mathrm{MHz}, \mathrm{CDCl}_{3}, \delta: 188.2(\mathrm{COSe}) ; 162.1\left(\mathrm{~d}, J_{C 4(F)-F}=259.9 \mathrm{~Hz}, \mathrm{C}_{4 \mathrm{Ph}(\mathrm{F})}\right) ; 134.3\right.$ $\left(\mathrm{d}, J_{1-F}=3.7 \mathrm{~Hz}, \mathrm{C}_{1 \mathrm{Ph}}\right) ; 130.3\left(\mathrm{~d}, J_{2-\mathrm{F}}=1.3 \mathrm{~Hz}, \mathrm{C}_{2 \mathrm{Ph}}\right) ; 128.1\left(\mathrm{~d}, J_{6-F}=8.7 \mathrm{~Hz}, \mathrm{C}_{6 \mathrm{Ph}}\right) ; 123.0$ $\left(\mathrm{d}, J_{3-F}=18.6 \mathrm{~Hz}, \mathrm{C}_{3 \mathrm{Ph}(\mathrm{Cl})}\right) ; 117.7\left(\mathrm{~d}, J_{5-F}=22.2 \mathrm{~Hz}, \mathrm{C}_{5 \mathrm{Ph}}\right) ; 116.9(\mathrm{CN})$; and $5.9\left(\mathrm{SeCH}_{2}\right)$. Elemental analysis for $\mathrm{C}_{9} \mathrm{H}_{5} \mathrm{ClFNOSe}$, calculated/found (\%): C: 39.09/39.12; H: 1.82/1.89; and N: 5.06/5.03. The acyl chloride (3-chloro-4-fluorobenzoyl chloride) was synthesized from 3-chloro-4-fluorobenzoic acid $(9.426 \mathrm{~g}, 50 \mathrm{mmol})$ and thionyl chloride $(35 \mathrm{~mL}$, excess), obtaining $9.602 \mathrm{~g}$ of the acyl chloride $(99.5 \%$ yield). Complete yield of the full synthetic route is then $40 \%$. 


\subsubsection{Se-(Cyanomethyl) 3,5-Bis(trifluoromethyl)benzoselenoate (N7)}

The reagents used: sodium borohydride $(0.197 \mathrm{~g}, 5.22 \mathrm{mmol})$, grey selenium $(0.197 \mathrm{~g}$,

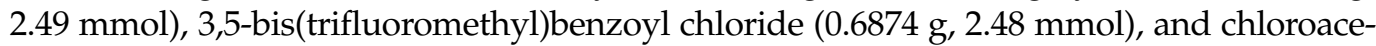
tonitrile $(0.191 \mathrm{~g}, 0.16 \mathrm{~mL}, 2.53 \mathrm{mmol})$. The final compound precipitated as a white solid powder that was isolated by filtration and washed with water, rendering $209 \mathrm{mg}(23 \%)$. MW: 360.12. Mp: $63-65{ }^{\circ} \mathrm{C}$. DIP-MS $m / z$ (abundance \%): 75.00 (3); 125.00 (2); 144.00 (5); 192.90 (2); 193.95 (4); 213.00 (45); 221.95 (2); and 241.00 (100). IR $\left(\mathrm{KBr}, \mathrm{cm}^{-1}\right)$ : 3098, 3029, $3004\left(\mathrm{~m}, \mathrm{C}-\mathrm{H}_{\mathrm{Ar}}\right) ; 2948\left(\mathrm{~s}, \mathrm{C}-\mathrm{H}_{\mathrm{Alk}}\right) ; 2246(\mathrm{~m}, \mathrm{C} \equiv \mathrm{N}) ; 1687(\mathrm{~s}, \mathrm{C}=\mathrm{O})$; and 1616, 1462 $\left(\mathrm{m}, \mathrm{C}-\mathrm{C}_{\mathrm{Ar}}\right) .{ }^{1} \mathrm{H}-\mathrm{NMR}\left(400 \mathrm{MHz}, \mathrm{CDCl}_{3}\right) ; \delta: 8.28\left(\mathrm{~s}, 2 \mathrm{H}, \mathrm{H}_{2 \mathrm{Ph}}+\mathrm{H}_{6 \mathrm{Ph}}\right) ; 8.17\left(\mathrm{~s}, 1 \mathrm{H}, \mathrm{H}_{4 \mathrm{Ph}}\right)$; and $3.79\left(\mathrm{~s}, 2 \mathrm{H}, \mathrm{SeCH}_{2}\right) .{ }^{13} \mathrm{C}-\mathrm{NMR}\left(101 \mathrm{MHz}, \mathrm{CDCl}_{3} ; \delta: 188.9(\mathrm{CO}) ; 139.0\left(\mathrm{C}_{1 \mathrm{Ph}}\right) ; 133.3\right.$ $\left(\mathrm{q}, J_{3-C F 3(3), 5-C F 3(5)}=34.5 \mathrm{~Hz}, \mathrm{C}_{3 \mathrm{Ph}}+\mathrm{C}_{5 \mathrm{Ph}}\right) ; 127.9\left(\mathrm{p}, J_{4-C F 3(3), 4-\mathrm{CF} 3(5)}=3.5 \mathrm{~Hz}, \mathrm{C}_{4 \mathrm{Ph}}\right) ; 127.4(\mathrm{q}$, $\left.J_{2-C F 3(3), 6-C F 3(5)}=2.9 \mathrm{~Hz}, \mathrm{C}_{2 \mathrm{Ph}_{2}}+\mathrm{C}_{6 \mathrm{Ph}}\right) ; 122.6\left(\mathrm{q}, J_{\mathrm{C}(\mathrm{CF} 3)-\mathrm{F}}=273.3 \mathrm{~Hz}, \mathrm{C}_{\mathrm{CF} 3(3)}+\mathrm{C}_{\mathrm{CF} 3(5)}\right) ; 116.4(\mathrm{CN}) ;$ and $6.4\left(\mathrm{SeCH}_{2}\right)$. Elemental analysis for $\mathrm{C}_{11} \mathrm{H}_{5} \mathrm{~F}_{6} \mathrm{NOSe}$, calculated/found (\%): $\mathrm{C}: 36.69 / 36.61$; H: 1.40/1.43; and N: 3.89/3.89. The acyl chloride (3,5-bis(trifluoromethyl)benzoyl chloride) was obtained from 3,5-bis(trifluoromethyl)benzoic acid $(12.906 \mathrm{~g}, 50 \mathrm{mmol}$ ) and thionyl chloride ( $35 \mathrm{~mL}$, excess), obtaining $7.422 \mathrm{~g}$ of the acyl chloride (54\% yield). Complete yield of the full synthetic route is then $13 \%$.

\subsection{Reagents, Medias, and Chemicals Used in the Biological Evaluation}

Analytical grade (to enable its use without further purification) rhodamine 123 (R123); sodium dodecyl sulfate (SDS); 3-(4.5-dimethylthiazol-2-yl)-2.5-diphenyltetrazolium bromide (MTT); Dulbecco's Modified Eagle's medium-high glucose (DMEM) with 10\% fetal bovine serum (FBS) and 1\% penicillin-streptomycin mixture; resazurin sodium salt; trypsinEDTA solution; allantoin; and tariquidar and dimethyl sulfoxide (DMSO) were acquired at Sigma-Aldrich (St. Louis, MO, USA). Doxorubicin hydrochloride was acquired from Teva Pharmaceuticals (Petah Tikva, Israel). Eagle's Minimal Essential Medium (EMEM, SigmaAldrich) containing $4500 \mathrm{mg} / \mathrm{L}$ glucose, supplemented with a non-essential amino acid (NEAA) mixture (Sigma-Aldrich); a selection of vitamins and 10\% heat-inactivated FBS; 2 mM L-glutamine (Sigma-Aldrich); 1 mM Na-pyruvate (Sigma-Aldrich); nystatin (SigmaAldrich); a penicillin-streptomycin mixture at concentrations of $100 \mathrm{U} / \mathrm{L}$ and $10 \mathrm{mg} / \mathrm{L}$; RPMI 1640 medium (Sigma-Aldrich), supplemented with 10\% FBS; 2 mM L-glutamine; 1 mM Na-pyruvate; $100 \mathrm{mM}$ HEPES (Sigma-Aldrich); nystatin; and a penicillin-streptomycin mixture at concentrations of $100 \mathrm{U} / \mathrm{L}$ and $10 \mathrm{mg} / \mathrm{L}$ were used in the biological evaluation. Pgp-Glo ${ }^{\mathrm{TM}}$ Assay Systems (Promega), and an Annexin V-FITC Apoptosis Detection Kit were used (Calbiochem, EMD Biosciences. Inc. La Jolla, CA, USA).

\subsection{Preparations of Compounds for Biological Assays}

The fifteen selenoesters (Figure 1) evaluated in this work, whose synthesis and characterization have been described above, were dissolved in dimethyl sulfoxide (DMSO) to obtain stock solutions with a $10 \mathrm{mM}$ concentration to perform their biological evaluation.

\subsection{Cell Lines and Their Maintenance}

Three cell lines have been used in this study: the doxorubicin-sensitive Colo 205 (CCL222, ATCC, Manassas, VA, USA) human colonic adenocarcinoma cell line; the multidrug resistant Colo 320/MDR-LRP expressing P-gp (MDR1)-LRP (CCL-220.1, ATCC) human colonic adenocarcinoma cell line; and the MRC-5 human embryonal lung fibroblast cell line (CCL-171, ATCC). The colon adenocarcinoma cell lines were purchased from LGC Promochem (Teddington, UK), and the MRC-5 cell line was purchased from Sigma-Aldrich (Merck KGaA, Darmstadt, Germany). Their culture conditions are as follows: Colo 205 (CCL-222, ATCC) and Colo 320/MDR-LRP expressing P-gp (MDR1)-LRP (CCL-220.1, ATCC) human colon adenocarcinoma cell lines were cultured in RPMI 1640 medium, supplemented with $10 \%$ FBS, $2 \mathrm{mM}$ L-glutamine, $1 \mathrm{mM}$ Na-pyruvate, and $100 \mathrm{mM}$ HEPES. The cell lines were incubated at $37{ }^{\circ} \mathrm{C}, 5 \% \mathrm{CO}_{2}$, and $95 \%$ air atmosphere. The semi-adherent human colon cancer cells were detached with a Trypsin-Versene (EDTA) solution for $5 \mathrm{~min}$ 
at $37^{\circ} \mathrm{C}$. MRC-5 human embryonal lung fibroblast cells were cultured in EMEM containing $4.5 \mathrm{~g} / \mathrm{L}$ of glucose and supplemented with a non-essential amino acid mixture, a selection of vitamins, and $10 \%$ FBS. The cell lines were incubated at $37{ }^{\circ} \mathrm{C}, 5 \% \mathrm{CO}_{2}$, and $95 \%$ air atmosphere.

The activity of selenoesters was tested on several cancer cell lines, including HepG2 (hepatocellular carcinoma, CCL-23TM, ATCC); HeLa (cervical adenocarcinoma, CCL-2TM, ATCC); B16 (skin melanoma, CCL-6322TM, ATCC); and a non-cancerous cell line, HDF (human dermal fibroblasts, Sigma-Aldrich). HepG2, HeLa, B16, and HDF cell lines were cultivated in EMEM medium supplemented with 10\% FBS, 2 mM L-glutamine, and 1\% penicillin-streptomycin mixture. The HaCaT cell line was cultivated in DMEM medium supplemented with $10 \%$ FBS and $1 \%$ penicillin-streptomycin mixture. All of the cells were cultivated in a $\mathrm{CO}_{2}$ incubator $\left(5 \% \mathrm{CO}_{2}, 37^{\circ} \mathrm{C}\right)$. Twice per week, the cell lines were passaged according to a standardized protocol with a trypsin-EDTA solution.

Wound healing activity was realized using human keratinocyte (HaCaT, Thermo Fisher Scientific, Waltham, MA, USA).

\subsection{Cytotoxicity}

The effects of increasing concentrations of the compounds on cell growth were tested in 96-well flat-bottomed microtiter plates. Moreover, $10^{4}$ of human colonic adenocarcinoma cells in $100 \mu \mathrm{L}$ of the medium (RPMI-1640) were added to each well, except for the medium control wells. The adherent human embryonic lung fibroblast cell line was seeded in the EMEM medium for $4 \mathrm{~h}$ before the assay. The two-fold serial dilutions of the compounds were made in a separate plate $(0.19-100 \mu \mathrm{M})$, and then transferred to the plates containing the adherent corresponding cell line. Culture plates were incubated at $37^{\circ} \mathrm{C}$ for $24 \mathrm{~h}$. At the end of the incubation period, $20 \mu \mathrm{L}$ of MTT (thiazolyl blue tetrazolium bromide) solution (from a $5 \mathrm{mg} / \mathrm{mL}$ stock solution) were added to each well. After incubation at $37^{\circ} \mathrm{C}$ for $4 \mathrm{~h}, 100 \mu \mathrm{L}$ of sodium dodecyl sulfate (SDS) solution (10\% SDS in $\left.0.01 \mathrm{M} \mathrm{HCl}\right)$ was added to each well, and the plates were further incubated at $37^{\circ} \mathrm{C}$ overnight. Cell growth was determined by measuring the optical density (OD) at $540 \mathrm{~nm}$ (ref. $630 \mathrm{~nm}$ ) with a Multiscan EX ELISA reader (Thermo Labsystems, Cheshire, WA, USA). Inhibition of cell growth was expressed as $\mathrm{IC}_{50}$ values, defined as the inhibitory dose that reduces the growth of the cells exposed to the tested compounds by $50 \%$. $\mathrm{IC}_{50}$ values and the SD of triplicate experiments were calculated by using GraphPad Prism software version 5.00 for Windows, with a non-linear regression curve fit (GraphPad Software, San Diego, CA, USA; www.graphpad.com, accessed on 12 July 2021). Doxorubicin (from a $2 \mathrm{mg} / \mathrm{mL}$ stock solution, Teva Pharmaceuticals) was used as a positive control. The solvent (DMSO) did not have any effect on the cell growth in the tested concentrations.

The selectivity indexes (SI) were calculated as the ratio of the $\mathrm{IC}_{50}$ value in the nontumour cells and the $\mathrm{IC}_{50}$ in the cancer cell lines. The compound's activity towards cancer cells is considered as strongly selective if the selectivity index (SI) value is higher than 6, moderately selective if $3<\mathrm{SI}<6$, slightly selective if $1<\mathrm{SI}<3$, and non-selective if SI is lower than 1 [16].

\subsection{Checkerboard Combination Assay}

A checkerboard microplate method was applied to study the effect of drug interactions between the selenocompounds and the chemotherapeutic drug doxorubicin on resistant Colo 320 colon adenocarcinoma cells expressing the ABCB1 transporter. Results were expressed in terms of the combination index (CI) values at $50 \%$ growth inhibition $\left(E_{50}\right)$, which were determined by using CompuSyn software to plot 4 or 5 data points for each ratio. CI values were calculated by means of the median-effect equation, where $\mathrm{CI}<1, \mathrm{CI}=1$, and $\mathrm{CI}>1$ represent synergism, an additive effect (or no interaction), and antagonism, respectively (Table 1 ).

To perform the experiment, the dilutions of doxorubicin were made in a horizontal direction in $100 \mu \mathrm{L}$, and the dilutions of the Se-compounds were made vertically in the 
microtiter plate in a volume of $50 \mu \mathrm{L}$. The cells were re-suspended in the culture medium and distributed into each well in $50 \mu \mathrm{L}$ portions containing 6000 cells. The plates were incubated for $72 \mathrm{~h}$ at $37^{\circ} \mathrm{C}$ in a $\mathrm{CO}_{2}$ incubator. The cell growth rate was determined after MTT staining. At the end of the incubation period, $20 \mu \mathrm{L}$ of MTT solution was added to each well. After incubation at $37^{\circ} \mathrm{C}$ for $4 \mathrm{~h}, 100 \mu \mathrm{L}$ of SDS was added to each well and the plates were further incubated at $37^{\circ} \mathrm{C}$ overnight. The optical density (OD) was measured at 540/630 $\mathrm{nm}$ with the Multiscan EX ELISA reader.

Table 1. Type of interactions based on the combination indexes.

\begin{tabular}{cccc}
\hline Combination Index $(\mathbf{C I})$ & Type of Interaction & Combination & Type of Interaction \\
\hline $0-0.1$ & very strong synergism & $0.9-1.1$ & additive effect \\
\hline $0.1-0.3$ & strong synergism & $1.1-1.2$ & slight antagonism \\
\hline $0.3-0.7$ & synergism & $1.2-1.45$ & $\begin{array}{c}\text { moderate antagonism } \\
\text { antagonism }\end{array}$ \\
\hline $0.7-0.85$ & moderate synergism & $3.45-3.3$ & strong antagonism \\
\hline $0.85-0.9$ & slight synergism & $>10$ & very strong antagonism \\
\hline
\end{tabular}

\subsection{ABCB1 Inhibition in the Presence of Selenoesters}

The inhibition of the ABCB1 multidrug efflux pump ABCB1 by the tested compounds was evaluated using flow cytometry, measuring the retention of rhodamine 123 by ABCB1 (P-glycoprotein) in Colo 320 colonic adenocarcinoma cells. Briefly, the cell number of colonic adenocarcinoma cells were adjusted to $2 \times 10^{6}$ cells $/ \mathrm{mL}$, re-suspended in serumfree RPMI-1640 medium in the case of colonic adenocarcinoma cells, and distributed in $0.5 \mathrm{~mL}$ aliquots into Eppendorf centrifuge tubes. The tested compounds were added at different concentrations ( 0.2 and $2 \mu \mathrm{M}$; from 1 and $10 \mathrm{mM}$ stock solutions, respectively), and the samples were incubated for $10 \mathrm{~min}$ at room temperature. Tariquidar was applied as the positive control $(0.2 \mu \mathrm{M}$ final concentration), and DMSO was used as the solvent control (at $2 v / v \%)$. Next, $10 \mu \mathrm{L}(5.2 \mu \mathrm{M}$ final concentration) of the fluorochrome rhodamine 123 (Sigma, St. Louis, MO, USA) was added to the samples and the cells were incubated for $20 \mathrm{~min}$ at $37^{\circ} \mathrm{C}$. After the incubation period, the cells were washed twice and re-suspended in $0.5 \mathrm{~mL}$ PBS for analysis. The fluorescence of the gated cell population was measured with a Partec CyFlow ${ }^{\circledR}$ flow cytometer (Partec, Münster, Germany). The percentage of the mean fluorescence intensity was calculated for the treated MDR cells as compared with the untreated cells. The results were obtained from a representative flow cytometry experiment in which at least 20,000 individual cells of the overall population were evaluated for the rhodamine 123 retained inside the cells. The fluorescence activity ratio (FAR) was calculated based on the following equation, which relates the measured fluorescence values:

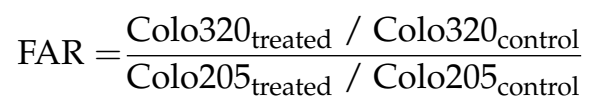

\subsection{P-gp ATPase Activity Assay}

P-glycoprotein ATPase activity was determined using the Pgp-Glo ${ }^{\mathrm{TM}}$ Assay System (Promega, WI, USA). The assay was performed according to the manufacturer's instructions. Next, $20 \mu \mathrm{L}$ of recombinant human P-gp membranes $(1.25 \mathrm{mg} / \mathrm{mL})$ were incubated for $5 \mathrm{~min}$ in $20 \mu \mathrm{L}$ of the Pgp-Glo ${ }^{\mathrm{TM}}$ assay buffer at $37^{\circ} \mathrm{C}$. Compounds were tested at $25 \mu \mathrm{M}$. Sodium orthovanadate $\left(\mathrm{Na}_{3} \mathrm{VO}_{4}, 0.25 \mathrm{mM}\right)$ was applied as an inhibitor control, and verapamil was used as a substrate control $(0.5 \mathrm{mM})$. DMSO at $2 \%$ was applied as a solvent control. The reaction was initiated by adding $10 \mu \mathrm{L}$ of $25 \mathrm{mM} \mathrm{MgATP}$, and incubated at $37^{\circ} \mathrm{C}$ for $40 \mathrm{~min}$. The reaction was stopped after adding $50 \mu \mathrm{L}$ of ATP Detection Reagent. Then, the samples and controls were incubated at room temperature for $20 \mathrm{~min}$. The emitted luciferase-generated luminescent signal was measured in a CLARIOstar Plus 
plate reader (BMG Labtech, UK) at $580 \mathrm{~nm}$. The relative ATPase activity was calculated based on the ratio between the luminescence measured of the P-gp ATPase activity of each compound and the basal P-gp ATPase activity according to the following equation:

$$
\text { Relative ATPase activity }=\frac{\mathrm{Lum}_{\text {treated }}-\mathrm{Lum}_{\text {untreated }}}{\mathrm{Lum}_{\mathrm{Na}_{3} \mathrm{VO}_{4}}-\mathrm{Lum}_{\text {untreated }}}
$$

The effect of the tested compounds was evaluated according to the instructions of the manufacturer (Table 2).

Table 2. Evaluation of Pgp ATPase activity.

\begin{tabular}{cc}
\hline$\Delta R L U_{T C}>\Delta R L U_{\text {basal }}$ & the tested compound is a stimulator of Pgp ATPase activity \\
\hline$\Delta R L U_{T C}=\Delta R L U_{\text {basal }}$ & the tested compound has no effect on Pgp ATPase activity \\
\hline$\Delta R L U_{T C}<\Delta R L U_{\text {basal }}$ & the tested compound is an inhibitor of Pgp ATPase activity \\
\hline
\end{tabular}

$\overline{\mathrm{RLU}}=$ Relative Light Unit; TC = tested compound. The difference in luminescent signal between $\mathrm{Na}_{3} \mathrm{VO}_{4}$-treated samples and untreated samples represents the basal Pgp ATPase activity.

\subsection{Apoptosis}

The assay was carried out using an Annexin V-FITC Apoptosis Detection Kit Cat. No. PF 032 from Calbiochem (EMD Biosciences. Inc. La Jolla. CA), according to the manufacturer's instructions. The concentration of the Colo 320 cell suspension was adjusted to approximately $1 \times 10^{6}$ cells $/ \mathrm{mL}$. The cell suspension was distributed into $1 \mathrm{~mL}$ aliquots $\left(1 \times 10^{6}\right.$ cells) into a 24 -well microplate and incubated overnight at $37^{\circ} \mathrm{C}$, with $5 \% \mathrm{CO}_{2}$. On the following day, the medium was removed and a fresh medium was added to the cells. The cells were incubated in the presence of Se-compounds for $3 \mathrm{~h}$ at $37^{\circ} \mathrm{C}$. The concentration for the apoptosis induction $(2 \mu \mathrm{M})$ was selected based on previous cytotoxicity results ( $\mathrm{IC}_{50}$ values). Moreover, $12 \mathrm{H}$-benzo $(\alpha)$ phenothiazine (M627) was used as positive control at $20 \mu \mathrm{M}$ final concentration. After a $3 \mathrm{~h}$ induction period, the culture medium was removed, the cells were washed with PBS, and a fresh medium was added to the cells. The 24 -well plates were incubated overnight at $37{ }^{\circ} \mathrm{C}$, with $5 \% \mathrm{CO}_{2}$. After the incubation, the supernatant was collected in a microfuge tube and $200 \mu \mathrm{L}$ of 0.25 trypsin (Trypsin-Versene) was added to the wells until the cells detached from the surfaces of the wells. The cells were centrifuged at $2000 \times g$ for $2 \mathrm{~min}$ at room temperature, the supernatant was removed, and the cells were re-suspended in fresh serum-free medium. After this procedure, apoptosis assay was carried out according to the rapid protocol of the kit using Annexin V-FITC and propidium iodide staining. The fluorescence was analysed immediately using a ParTec CyFlow ${ }^{\circledR}$ flow cytometer (Partec, Münster, Germany), and the results were obtained from a representative flow cytometry experiment in which at least 20,000 individual cells of the overall population in a sample were evaluated. The data were analysed by FlowJo ${ }^{\mathrm{TM}}$ software (BD Biosciences, San Jose, NJ, USA).

\subsection{Wound Healing Assay}

Wound healing was measured in vitro by scratch assay, as the migration rate of the cells to close the gap was created, as described by Ling et al. [19], with slight modifications. Briefly, HaCaT cells at the concentration of $5 \times 10^{5}$ cells were seeded into 12-well plates and incubated at $37{ }^{\circ} \mathrm{C}$ and $5 \% \mathrm{CO}_{2}$ until the cells formed a uniform layer. After layer formation, the cells were washed with PBS and then scratched with a sterile micropipette tip. The debris was removed by further gentle washing with PBS, then the samples ( $\left.\mathrm{IC}_{10}\right)$, along with the positive control (Allantoin, $50 \mu \mathrm{g} / \mathrm{mL}$ ), were mixed with DMEM and added directly to the cells. The images of the scratch area were captured using an inverted microscope in $4 \times$ magnification at different time intervals $(0 \mathrm{~h}, 24 \mathrm{~h}, 48 \mathrm{~h})$ and evaluated using ImageJ software (National Institutes of Health, Bethesda, MD, USA). All of the experiments were performed in four replicates, and a minimum of five measurements were considered from each image captured. Dean-Dixon's test was used to remove the 
outliners in the values measured, and further statistical significances between the groups were established by $t$-test (Excel, Microsoft Office, Redmond, WA, USA). The wound closure was calculated as the ratio of the gap size at the beginning minus the gap size at the evaluated time.

\section{Results}

\subsection{Synthesis and Characterization of the Compounds}

The ketone derivatives have been designed as derivatives of the potent oxoselenoesters previously reported [15-18], attempting to improve their activity and selectivity. The compounds published in these works were the Se-(2-oxopropyl) 4-clorobenzoselenoate (compound 9), the Se-(3,3-dimethyl-2-oxobutyl) 4-clorobenzoselenoate (compound 10), and the Se-(3,3-dimethyl-2-oxobutyl) 3,5-dimethoxybenzoselenoate (compound 11). Cyanoselenoesters have been designed as a variation of the ones included in a previous patent of the group [18], with the same improvement aim of the activity showed by the initial cyanoselenoesters included in this patent.

Seeing the noteworthy activity in previous works [15-18] shown by derivatives that contain halogens, we have considered herein different halogenated substituents (bromine, fluorine, trifluoromethyl, chlorine), as well their polysubstitution, to determine which ones enhanced the biological effects. Additionally, compounds with a thiophene ring or with a 4-tert-butyl substituent are included to evaluate the activity of compounds that contain heterocycles or electron-donating substituents, respectively.

The new 15 compounds reported in this work were pure and chemically stable at room temperature. All of them were pure in the Elemental Analysis, following the threshold of a maximum variation of $\pm 0.4 \%$ for every element tested $(C, H, N, S)$. The yield is markedly affected by the state of the matter of the product: generally, liquid compounds require purification by column chromatography, and its handling is more troublesome, which is reflected in the yields. Typically, solid compounds showed yields in the range from $40 \%$ to $50 \%$, with the exception of compound K6 (18\%); whereas liquid compounds have lower yields, from $7 \%$ to $29 \%$. As mentioned above, the synthetic route consisted of three steps: an initial in situ preparation of the selenating agent, and the attack of this agent to the suitable benzoyl or thienyl chloride to form an acyl selenide salt that exerts a nucleophilic attack over a suitable alkyl halide (chloroacetone for derivatives $\mathbf{K 1 - K 8}$ and chloroacetonitrile for compounds N1-N7). A few derivatives needed a preliminary step to prepare the required benzoyl chloride from the respective benzoic acid. These compounds were: K5 and N5 from 3-(trifluoromethyl)benzoic acid; K6 and N6 from 3-chloro-4-fluorobenzoic acid; K8 from 2,4,5-trifluorobenzoic acid; and N7 from 3,5-bis(trifluoromethyl)benzoic acid.

Compounds $\mathrm{K1}-\mathrm{K} 8$ have, as a common feature the $-\mathrm{COSeCH}_{2} \mathrm{COCH}_{3}$ moiety bound to the phenyl or thienyl ring. In ${ }^{1} \mathrm{H}-\mathrm{NMR}$, the $\mathrm{CH}_{2}$ appears as a singlet accompanied by two small satellite peaks (due to the influence of the adjacent selenium atom) in the range $3.89-3.96 \mathrm{ppm}$, and the $\mathrm{CH}_{3}$ in the range $2.33-2.36 \mathrm{ppm}$. Alternatively, in ${ }^{13} \mathrm{C}-\mathrm{NMR}$, the $\mathrm{CH}_{2}$ again appears as a singlet accompanied by two small satellite peaks (due to the influence of the adjacent selenium atom) in the range 34.5-36.0 ppm, and the $\mathrm{CH}_{3}$ in the range $28.6-29.2 \mathrm{ppm}$. The carbonyls appear in different ranges in ${ }^{13} \mathrm{C}-\mathrm{NMR}$ : there is a higher and narrower range of 203.1-204.1 ppm for the ketone carbonyl, and a less displaced and wider range of 183.0-193.3 ppm for the carbonyl group of the selenoester. The width of the ranges is logical, as the width increases when the substituted phenyl ring is closer. Similarly, in the case of the N1-N7 derivatives, the common structural moiety is the $-\mathrm{COSeCH}_{2} \mathrm{CN}$ bound to the phenyl or thienyl ring. The $-\mathrm{CH}_{2}$ bound to the selenium and to the cyano group, which appears in ${ }^{1} \mathrm{H}-\mathrm{NMR}$ in the range from 3.70 to $3.79 \mathrm{ppm}$. This carbon is seen in ${ }^{13} \mathrm{C}-\mathrm{NMR}$ at a very low displacement (ranging from 5.5 to $7.0 \mathrm{ppm}$ ). The $-\mathrm{CN}$ group is observed in a very narrow range (116.4-117.2 ppm), and the -COSe appears at a slightly lower range than the -COSe of the ketone derivatives (180.4-191.2). The remaining signals depend on the ring (thienyl for $\mathbf{K 1}$ and N1, and phenyl for the remaining compounds), and on the substituents (and their position) bound to the phenyl 
ring. For a few compounds (K1, K3, K7, K8, N1, and N5), bidimensional NMR spectra as ${ }^{1} \mathrm{H}_{-}{ }^{1} \mathrm{H}$ COSY, ${ }^{1} \mathrm{H}_{-}{ }^{13} \mathrm{C}$ HSQC, and ${ }^{1} \mathrm{H}_{-}^{13} \mathrm{C}$ HMBC have been recorded to help in the correct assignment of the signals. Of these bidimensional spectra, $\mathrm{HMBC}$ was of particular interest, as the spectra obtained proof of the $-\mathrm{COSeCH}_{2} \mathrm{CN}$ or the $-\mathrm{COSeCH}_{2} \mathrm{COCH}_{3}$ thanks to the long-distance interactions between the different atoms involved (see spectra in Supplementary: Figures S1H, S3H, S7G, S8I, S9H, and S13I).

In mass spectrometry, the most abundant ion is always the product of the breakage of the carbon-selenium bond of the selenoester, releasing as a positive ion the acyl cation (which is the peak with $100 \%$ of abundance) and an anion that includes the selenium atom. From this acyl cation, different cations are also observed, including the release of the thiophenium cation (compounds $\mathbf{K} \mathbf{1}$ and N1) or the appropriate phenyl cation. The latest overcomes different releases (for example: $\mathrm{HF}, \mathrm{HCl}$, and $\mathrm{HBr}$ ) depending on the substituents present at the ring. The easiness of the breakage of the C-Se bond implies that the molecular ion always has a very low abundance, and it was even not observed in four compounds (N3, N4, N6, and N7). Additionally, the molecular ion showed an abundance below $0.5 \%$ in 5 compounds of the 11 remaining. Interestingly, abundances of the molecular ion were always higher at ketone-selenoesters (from $0 \%$ to $2 \%$ ) than at cyanoselenoesters (not observed or below $0.5 \%$ ). Perhaps this fact may point out that, at least in the conditions of the ionization chamber, the first are more stable.

Regarding infrared spectroscopy, all ketone-selenoesters showed two peaks in the range of 1720-1650, where the $\mathrm{C}=\mathrm{O}$ stretch of carbonyl compounds are usually observed. The carbonyl of the selenoester had a lower (from 1650 to $1679 \mathrm{~cm}^{-1}$ ), and a wider range than the one experimentally determined for the carbonyl of the ketone (from 1697 to $1712 \mathrm{~cm}^{-1}$ ), due to the fact that it was the carbonyl group in the molecule which is closer to the substituents than the ketone carbonyl. The exception was the compound $\mathbf{K} 4$, which produced a wider overlapping signal that incorporated the two carbonyl compounds. Alternatively, the IR spectra of the nitrile derivatives have, as a common feature, the $\mathrm{C} \equiv \mathrm{N}$ stretch and the $\mathrm{C}=\mathrm{O}$ stretch of the selenoester. They appeared in the ranges $2239-2247 \mathrm{~cm}^{-1}$ and $1662-1706 \mathrm{~cm}^{-1}$, respectively.

\subsection{Cytotoxicity}

Based on the obtained results, ketone-selenoesters showed a potent cytotoxic effect on both sensitive and resistant colon cancer cell lines, where the $\mathrm{IC}_{50}$ values were between 1 and $4 \mu \mathrm{M}$ on both cell lines. In addition, similar toxic activity was observed on MRC-5 normal lung fibroblast cells, indicating that ketone-selenoesters have no selectivity towards cancer cells. Cyanoselenoesters showed no effect on the MRC- 5 cell line ( $\mathrm{IC}_{50}$ was more than $100 \mu \mathrm{M}$ ); however, they were very toxic on both colon cancer cell lines. Comparing the sensitive Colo $205\left(\mathrm{IC}_{50}: 1.98-2.96 \mu \mathrm{M}\right)$ to the resistant Colo 320 cells $\left(\mathrm{IC}_{50}\right.$ : 3.78-7.64 $\mu \mathrm{M}$ ), cyanoselenoesters were less potent on the resistant cells. Furthermore, both ketone- and cyanoselenoesters were more active on cancer cell lines than the positive control doxorubicin. Selectivity indexes (SI) were calculated as described above. Cyanoselenoesters showed high selectivity $(>6)$ in each case, and ketone-selenoesters showed moderate $(3<\mathrm{SI}<6)$ and slight $(1<\mathrm{SI}<3)$ selectivity, except K2 $(\mathrm{SI}<1)$. (Table 3).

Similar results were obtained in cancer cells from other locations, such as liver, cervix, and skin. Ketone-selenoesters showed the following range of $\mathrm{IC}_{50}$ values in these cancer cells (Table 4): from 2.2 to $4.3 \mu \mathrm{M}$ towards hepatocellular carcinoma (HepG2) cells, from 1.9 to $2.7 \mu \mathrm{M}$ towards cervical adenocarcinoma (HeLa) cells, and from 1.1 to $2.0 \mu \mathrm{M}$ towards skin murine melanoma (B16) cells. Regarding cyanoselenoesters, the observed range of $\mathrm{IC}_{50}$ values in the abovementioned cell lines were from 5.2 to $11.8 \mu \mathrm{M}$ towards HepG2 cells, from 1.3 to $5.2 \mu \mathrm{M}$ towards the HeLa cell line, and from 1.4 to $2.6 \mu \mathrm{M}$ towards B16 cells. SI values were again higher than 6 for all of the cyanoselenoesters in HepG2 and HeLa cells, indicating that all of the cyanoselenoesters were strongly selective towards the cancer cells in respect to MRC- 5 cells. In contrast, none of the ketone-selenoesters showed an SI value higher than three (moderately selective) for these two additional human cancer cells. 
Table 3. Cytotoxic effect of selenocompounds on sensitive (Colo 205) and resistant (Colo 320) colon adenocarcinoma, and MRC-5 normal embryonal fibroblast cell lines and selectivity indexes (SI). Doxorubicin was used as a positive control.

\begin{tabular}{|c|c|c|c|c|c|c|c|c|}
\hline \multirow{2}{*}{ Cpds. } & \multicolumn{2}{|c|}{ Colo $205\left(\mathrm{IC}_{50} \mu \mathrm{M}\right)$} & \multicolumn{2}{|c|}{ Colo $320\left(\mathrm{IC}_{50} \mu \mathrm{M}\right)$} & \multicolumn{2}{|c|}{ MRC-5 $\left(\mathrm{IC}_{50} \mu \mathrm{M}\right)$} & \multirow{2}{*}{$\begin{array}{c}\text { SI } \\
\text { MRC-5/Colo } 205\end{array}$} & \multirow{2}{*}{$\begin{array}{c}\text { SI } \\
\text { MRC-5/Colo } 320\end{array}$} \\
\hline & Mean & SD & Mean & SD & Mean & SD & & \\
\hline K1 & 1.53 & \pm 0.46 & 1.47 & \pm 0.02 & 2.24 & \pm 0.29 & 1.46 & 1.52 \\
\hline K2 & 3.35 & \pm 0.58 & 2.38 & \pm 0.23 & 2.53 & \pm 0.40 & 0.76 & 1.06 \\
\hline $\mathrm{K} 3$ & 2.28 & \pm 0.05 & 2.15 & \pm 0.03 & 2.86 & \pm 0.36 & 1.25 & 1.33 \\
\hline K4 & 1.05 & \pm 0.04 & 1.48 & \pm 0.06 & 3.63 & \pm 0.37 & 3.46 & 2.45 \\
\hline K5 & 2.14 & \pm 0.08 & 2.17 & \pm 0.27 & 3.11 & \pm 3.93 & 1.45 & 1.43 \\
\hline K6 & 2.10 & \pm 0.02 & 2.10 & \pm 0.05 & 3.62 & \pm 0.41 & 1.73 & 1.72 \\
\hline K7 & 2.69 & \pm 0.07 & 2.57 & \pm 0.15 & 3.72 & \pm 0.17 & 1.38 & 1.45 \\
\hline K8 & 2.24 & \pm 0.16 & 2.37 & \pm 0.11 & 2.50 & \pm 0.06 & 1.12 & 1.05 \\
\hline N1 & 2.37 & \pm 0.27 & 7.64 & \pm 0.15 & $>100$ & - & $>6^{*}$ & $>6^{*}$ \\
\hline N2 & 2.96 & \pm 0.09 & 7.01 & \pm 0.69 & $>100$ & - & $>6^{*}$ & $>6^{*}$ \\
\hline N3 & 2.10 & \pm 0.06 & 4.37 & \pm 0.10 & $>100$ & - & $>6^{*}$ & $>6^{*}$ \\
\hline N4 & 1.97 & \pm 0.14 & 5.57 & \pm 0.23 & $>100$ & - & $>6$ * & $>6 *$ \\
\hline N5 & 2.10 & \pm 0.10 & 5.22 & \pm 0.08 & $>100$ & - & $>6^{*}$ & $>6^{*}$ \\
\hline N6 & 2.24 & \pm 0.07 & 5.19 & \pm 0.37 & $>100$ & - & $>6$ * & $>6$ * \\
\hline N7 & 1.98 & \pm 0.16 & 3.78 & \pm 0.23 & $>100$ & - & $>6 *$ & $>6 *$ \\
\hline Dox. & 3.46 & \pm 0.34 & 7.61 & \pm 0.29 & 2.73 & \pm 0.34 & 0.79 & 0.36 \\
\hline
\end{tabular}

Data are presented as the average of three measurements with the respective standard error of the mean. Dox. = doxorubicin. ${ }^{*}$ The derivatives were not toxic on normal human fibroblast (MRC-5). For this reason, the numeric value of selectivity could not be precisely determined; however, all derivatives proved to be selective.

Table 4. Cytotoxic effect of selenocompounds on hepatocellular carcinoma (HepG2), cervical adenocarcinoma (HeLa), and skin melanoma (B16) cell lines and selectivity indexes (SI).

\begin{tabular}{|c|c|c|c|c|c|c|c|c|c|}
\hline \multirow{2}{*}{ Cpds. } & \multicolumn{2}{|c|}{ HepG2 (IC $\left.{ }_{50} \mu \mathrm{M}\right)$} & \multicolumn{2}{|c|}{ HeLa $\left(I_{50} \mu \mathrm{M}\right)$} & \multicolumn{2}{|c|}{$\mathrm{B} 16\left(\mathrm{IC}_{50} \mu \mathrm{M}\right)$} & \multirow{2}{*}{$\begin{array}{c}\text { SI } \\
\text { MRC-5/HepG2 }\end{array}$} & \multirow{2}{*}{$\begin{array}{c}\text { SI } \\
\text { MRC-5/HeLa }\end{array}$} & \multirow{2}{*}{$\begin{array}{c}\text { SI } \\
\text { MRC-5/B16 }\end{array}$} \\
\hline & Mean & SD & Mean & SD & Mean & SD & & & \\
\hline K1 & 2.3 & \pm 0.2 & 2.5 & \pm 0.2 & 1.4 & \pm 0.1 & 1.6 & 1.5 & 2.6 \\
\hline $\mathrm{K} 2$ & 2.2 & \pm 0.215 & 2.5 & \pm 0.5 & 1.2 & \pm 0.1 & 1.1 & 1.0 & 2.1 \\
\hline K3 & 3.1 & \pm 0.2 & 2.1 & \pm 0.2 & 1.7 & \pm 0.2 & 0.7 & 1.1 & 1.3 \\
\hline K4 & 4.3 & \pm 0.1 & 1.9 & \pm 0.0 & 2.0 & \pm 0.2 & 0.7 & 1.5 & 1.4 \\
\hline K5 & 2.4 & \pm 0.2 & 2.5 & \pm 0.1 & 1.1 & \pm 0.1 & 1.3 & 1.2 & 2.8 \\
\hline K6 & 2.9 & \pm 0.3 & 2.3 & \pm 0.1 & 1.3 & \pm 0.1 & 1.3 & 1.6 & 2.8 \\
\hline K7 & 3.7 & \pm 0.3 & 2.7 & \pm 0.3 & 1.4 & \pm 0.1 & 1.0 & 1.4 & 2.7 \\
\hline K8 & 4.0 & \pm 0.2 & 2.0 & \pm 0.0 & 1.4 & \pm 0.1 & 0.6 & 1.3 & 1.8 \\
\hline N1 & 11.3 & \pm 0.9 & 2.0 & \pm 0.1 & 1.9 & \pm 0.4 & $>6^{*}$ & $>6^{*}$ & $>6^{*}$ \\
\hline N2 & 5.6 & \pm 0.3 & 5.2 & \pm 0.5 & 2.6 & \pm 0.4 & $>6^{*}$ & $>6^{*}$ & $>6^{*}$ \\
\hline N3 & 9.6 & \pm 0.9 & 2.4 & \pm 0.1 & 2.8 & \pm 0.5 & $>6^{*}$ & $>6 *$ & $>6^{*}$ \\
\hline N4 & 5.2 & \pm 0.3 & 2.5 & \pm 0.0 & 1.4 & \pm 0.3 & $>6^{*}$ & $>6^{*}$ & $>6^{*}$ \\
\hline N5 & 9.8 & \pm 0.6 & 2.5 & \pm 0.2 & 1.4 & \pm 0.3 & $>6^{*}$ & $>6^{*}$ & $>6^{*}$ \\
\hline N6 & 9.6 & \pm 0.4 & 1.3 & \pm 0.1 & 1.7 & \pm 0.3 & $>6^{*}$ & $>6 *$ & $>6^{*}$ \\
\hline N7 & 11.8 & \pm 1.2 & 2.1 & \pm 0.1 & 1.6 & \pm 0.4 & $>6^{*}$ & $>6 *$ & $>6^{*}$ \\
\hline
\end{tabular}

* The derivatives were not toxic on normal human fibroblast cells (MRC-5) up to $100 \mu \mathrm{M}$. For this reason, the numeric value of selectivity could not be precisely determined; however, all derivatives proved to be selective.

\subsection{Checkerboard Combination Assay}

The checkerboard combination assay is a widely used and convenient in vitro method for the assessment of drug interactions among various pharmacological agents. This program enables the calculation of the combination indices, and also allows the determination of the most effective ratios of combinational agents. Ketone- and cyanoselenoesters were combined with doxorubicin, and their interactions were determined after MTT staining. The data obtained were analysed using Calcusyn software.

Six ketone-selenoesters $(\mathbf{K} \mathbf{1}, \mathbf{K} 3, \mathbf{K} 4, \mathbf{K} 5, \mathbf{K} 6, \mathbf{K} 8)$ were found to interact synergistically with doxorubicin. Furthermore, $\mathbf{K} 5$ and $\mathbf{K} 6$ showed a synergistic interaction with doxorubicin in all ratios (Table 5). Additionally, a synergistic effect can be seen for five 
cyanoselenoesters (N1, N2, N3, N4, N7) (Table 6). The type of interaction in the combination studies was evaluated using the Chou-Talalay method for drug combination, which is based on the median-effect equation. The prerequisite for the calculation is the dose-effect curves for each drug alone. The combination of two drugs at a certain ratio behaves like a third drug to the cells. In this way, the parameters can be obtained for the mixture, just in case the of the single drugs, by using the automated median-effect plot with computer software. Applying this method, several ratios can be tested and different types of interactions can be obtained, allowing for a more precise description of the interaction of the compounds [20].

Table 5. Interaction of ketone-selenoesters with doxorubicin on MDR Colo 320 cells.

\begin{tabular}{|c|c|c|c|c|c|}
\hline Compounds & Starting Conc. $(\mu \mathrm{M})$ & Ratio * & $\mathrm{CI}$ at $\mathrm{ED}_{50}$ & $\mathrm{SD}( \pm)$ & Type of Interaction \\
\hline \multirow[t]{5}{*}{ K1 } & 5 & $0.6: 1$ & 2.6 & 0.73 & Antagonism \\
\hline & & $1.2: 1$ & 1.03 & 0.11 & Additive effect \\
\hline & & $2.4: 1$ & 0.94 & 0.09 & Additive effect \\
\hline & & $4.8: 1$ & 0.88 & 0.13 & Slight synergism \\
\hline & & $9.6: 1$ & 1.18 & 0.15 & Slight antagonism \\
\hline \multirow[t]{6}{*}{$\mathbf{K} 2$} & 6 & $0.7: 1$ & 1.77 & 0.22 & Antagonism \\
\hline & & $1.4: 1$ & 2.95 & 0.16 & Antagonism \\
\hline & & $2.8: 1$ & 1.2 & 0.22 & Slight antagonism \\
\hline & & $5.6: 1$ & 1.02 & 0.22 & Additive effect \\
\hline & & $11.2: 1$ & 1.5 & 0.27 & Antagonism \\
\hline & & $22.4: 1$ & 2.34 & 0.59 & Antagonism \\
\hline \multirow[t]{6}{*}{ K3 } & 6 & $0.7: 1$ & 1.32 & 0.8 & Moderate antagonism \\
\hline & & $1.4: 1$ & 0.37 & 0.15 & Synergism \\
\hline & & $2.8: 1$ & 0.73 & 0.1 & Moderate synergism \\
\hline & & $5.6: 1$ & 1.5 & 0.24 & Antagonism \\
\hline & & $11.2: 1$ & 1.3 & 0.07 & Moderate antagonism \\
\hline & & $22.4: 1$ & 1.72 & 0.08 & Antagonism \\
\hline \multirow[t]{6}{*}{ K4 } & 5 & $0.6: 1$ & 0.54 & 0.07 & Synergism \\
\hline & & $1.2: 1$ & 1.03 & 0.05 & Additive effect \\
\hline & & $2.4: 1$ & 1.1 & 0.05 & Additive effect \\
\hline & & $4.8: 1$ & 0.74 & 0.1 & Moderate synergism \\
\hline & & $9.6: 1$ & 0.85 & 0.06 & Slight synergism \\
\hline & & $19.2: 1$ & 0.97 & 0.12 & Additive effect \\
\hline \multirow[t]{6}{*}{ K5 } & 6 & $0.7: 1$ & 0.51 & 0.06 & Synergism \\
\hline & & $1.4: 1$ & 0.81 & 0.05 & Moderate synergism \\
\hline & & $2.8: 1$ & 0.55 & 0.04 & Synergism \\
\hline & & $5.6: 1$ & 0.58 & 0.02 & Synergism \\
\hline & & $11.2: 1$ & 0.64 & 0.02 & Synergism \\
\hline & & $22.4: 1$ & 0.68 & 0.06 & Synergism \\
\hline \multirow[t]{6}{*}{ K6 } & 6 & $0.7: 1$ & 0.51 & 0.06 & Synergism \\
\hline & & $1.4: 1$ & 0.81 & 0.05 & Moderate synergism \\
\hline & & $2.8: 1$ & 0.55 & 0.04 & Synergism \\
\hline & & $5.6: 1$ & 0.58 & 0.02 & Synergism \\
\hline & & $11.2: 1$ & 0.64 & 0.02 & Synergism \\
\hline & & 22.4:1 & 0.68 & 0.06 & Synergism \\
\hline \multirow[t]{6}{*}{$\mathbf{K} 7$} & 6 & $0.7: 1$ & 1.4 & 0.2 & Moderate antagonism \\
\hline & & $1.4: 1$ & 3.1 & 0.41 & Antagonism \\
\hline & & $2.8: 1$ & 1.36 & 0.2 & Moderate antagonism \\
\hline & & $5.6: 1$ & 1.3 & 0.07 & Moderate antagonism \\
\hline & & $11.2: 1$ & 2.8 & 0.15 & Antagonism \\
\hline & & $22.4: 1$ & 2.28 & 0.15 & Antagonism \\
\hline \multirow[t]{5}{*}{ K8 } & 6 & $0.7: 1$ & 0.12 & 0.09 & Strong synergism \\
\hline & & $1.4: 1$ & 2.4 & 0.62 & Antagonism \\
\hline & & 2.8:1 & 3.3 & 0.8 & Antagonism \\
\hline & & $5.6: 1$ & 2.01 & 0.97 & Antagonism \\
\hline & & 11.2:1 & 3.3 & 0.74 & Antagonism \\
\hline
\end{tabular}

* Ratio: the applied combination and the concentration of selenoester-doxorubicin combination. CI at ED50: combination index value (CI) at the $50 \%$ growth inhibition dose $\left(\mathrm{ED}_{50}\right)$. The most effective interactions (types of synergism) for each derivative are highlighted in bold. 
Table 6. Interaction of cyanoselenoesters with doxorubicin on MDR Colo 320 colon adenocarcinoma cells.

\begin{tabular}{|c|c|c|c|c|c|}
\hline Compounds & Starting Conc. $(\mu \mathrm{M})$ & Ratio * & $C I$ at $E D_{50}$ & $\mathrm{SD}( \pm)$ & Type of Interaction \\
\hline \multirow[t]{6}{*}{ N1 } & 15 & $1.7: 1$ & 1.9 & 0.2 & Antagonism \\
\hline & & $3.4: 1$ & 0.34 & 0.04 & Synergism \\
\hline & & $6.8: 1$ & 0.51 & 0.04 & Synergism \\
\hline & & $13.6: 1$ & 0.95 & 0.15 & Additive effect \\
\hline & & $27.2: 1$ & 0.56 & 0.09 & Synergism \\
\hline & & $54.4: 1$ & 0.21 & 0.21 & Strong synergism \\
\hline \multirow{7}{*}{ N2 } & & $54.4: 1$ & 0.21 & 0.21 & Strong synergism \\
\hline & 15 & $1.7: 1$ & 0.62 & 0.19 & Synergism \\
\hline & & $3.4: 1$ & 3.1 & 0.38 & Antagonism \\
\hline & & $6.8: 1$ & 0.58 & 0.03 & Synergism \\
\hline & & $13.6: 1$ & 1.36 & 0.05 & Moderate antagonism \\
\hline & & $27.2: 1$ & 2.8 & 0.12 & Antagonism \\
\hline & & $54.4: 1$ & 2.3 & 0.15 & Antagonism \\
\hline \multirow[t]{6}{*}{ N3 } & 10 & $1.2: 1$ & 1.7 & 0.44 & Antagonism \\
\hline & & $2.4: 1$ & 3.7 & 0.5 & Strong antagonism \\
\hline & & $4.8: 1$ & 0.85 & 0.1 & Moderate synergism \\
\hline & & $9.6: 1$ & 1.01 & 0.15 & Additive effect \\
\hline & & $19.2: 1$ & 1.19 & 0.17 & Slight antagonism \\
\hline & & 38.4:1 & 1.3 & 0.21 & Moderate antagonism \\
\hline \multirow[t]{6}{*}{ N4 } & 10 & $1.2: 1$ & 1.7 & 0.44 & Antagonism \\
\hline & & $2.4: 1$ & 3.7 & 0.5 & Strong antagonism \\
\hline & & $4.8: 1$ & 0.85 & 0.1 & Moderate synergism \\
\hline & & $9.6: 1$ & 1.01 & 0.15 & Additive effect \\
\hline & & $19.2: 1$ & 1.19 & 0.17 & Slight antagonism \\
\hline & & 38.4:1 & 1.3 & 0.21 & Moderate antagonism \\
\hline \multirow[t]{6}{*}{ N5 } & 10 & $1.2: 1$ & 5.7 & 1.6 & Strong antagonism \\
\hline & & $2.4: 1$ & 3.7 & 0.5 & Strong antagonism \\
\hline & & $4.8: 1$ & 1.2 & 0.95 & Slight antagonism \\
\hline & & $9.6: 1$ & 1.01 & 0.15 & Additive effect \\
\hline & & $19.2: 1$ & 1.19 & 0.17 & Slight antagonism \\
\hline & & $38.4: 1$ & 1.3 & 0.21 & Moderate antagonism \\
\hline \multirow[t]{6}{*}{ N6 } & 10 & $1.2: 1$ & 2.9 & 0.31 & Antagonism \\
\hline & & $2.4: 1$ & 4.5 & 0.5 & Additive effect \\
\hline & & $4.8: 1$ & 1.5 & 0.1 & Antagonism \\
\hline & & $9.6: 1$ & 1.78 & 0.21 & Antagonism \\
\hline & & $19.2: 1$ & 1.96 & 0.11 & Antagonism \\
\hline & & $38.4: 1$ & 1.96 & 0.105 & Antagonism \\
\hline \multirow[t]{6}{*}{ N7 } & 8 & 0.9:1 & 2.7 & 0.23 & Antagonism \\
\hline & & $1.8: 1$ & 5.3 & 1.02 & Strong antagonism \\
\hline & & $3.6: 1$ & 0.9 & 0.23 & Slight synergism \\
\hline & & $7.2: 1$ & 1.84 & 0.18 & Antagonism \\
\hline & & $14.4: 1$ & 1.89 & 0.29 & Antagonism \\
\hline & & $28.8: 1$ & 0.92 & 0.6 & Additive effect \\
\hline
\end{tabular}

* Ratio: the applied combination and the concentration of selenocompound-doxorubicin. $\mathrm{CI}$ at $\mathrm{ED}_{50}$ : combination index value (CI) at the $50 \%$ growth inhibition dose $\left(\mathrm{ED}_{50}\right)$. The most effective interactions (types of synergism) for each derivative are highlighted in bold.

\subsection{ABCB1 Inhibition in the Presence of Selenoesters}

The inhibition of the ABCB1 transporter was assessed by measuring the intracellular accumulation of its fluorescent substrate rhodamine 123 at 0.2 and $2 \mu \mathrm{M}$. Based on the flow cytometric evaluation, ketone-selenoesters inhibited the activity of the ABCB1 transporter, and the most potent derivatives were $\mathbf{K 1}, \mathbf{K} 2, \mathbf{K} 3, \mathbf{K} 7$, and $\mathbf{K} 8$, exhibiting a FAR value of $45.73,37.35,39.17,40.38$, and 36.09 at $2 \mu \mathrm{M}$, respectively. Even the less potent derivatives showed similar activity at $2 \mu \mathrm{M}$ : in the case of K5, the FAR value was 32.61. Furthermore, in the presence of K6, a FAR of 29.4 was obtained. The less active derivative was $\mathbf{K} 4$, with a 
FAR value of 4.99 at $2 \mu \mathrm{M}$. K3 and K8 were effective at $0.2 \mu \mathrm{M}$ and $2 \mu \mathrm{M}$ concentration (FAR at $0.2 ; \mu \mathrm{M}$ at 3.99 and 3.38 , respectively), while the other compounds were only effective at $2 \mu \mathrm{M}$ concentration (Figure 2). Cyanoselenoesters did not show ABCB1 modulating activity.

\begin{tabular}{cccccc}
\hline Compounds & $\begin{array}{c}\text { Concentration } \\
(\mu \mathrm{M})\end{array}$ & FSC & SSC & FL-1 & FAR \\
\hline K1 & 0.2 & 2144 & 989 & 2.5 & 1.37 \\
K2 & 2 & 1866 & 1067 & 83 & $\mathbf{4 5 . 7 3}$ \\
& 0.2 & 1916 & 1061 & 2.56 & 1.41 \\
K3 & 2 & 1945 & 1156 & 67.8 & $\mathbf{3 7 . 3 5}$ \\
& 0.2 & 1706 & 982 & 7.25 & $\mathbf{3 . 9 9}$ \\
K4 & 2 & 2040 & 1031 & 71.1 & $\mathbf{3 9 . 1 7}$ \\
& 0.2 & 1808 & 861 & 3.19 & 1.76 \\
K5 & 2 & 1830 & 1008 & 9.06 & $\mathbf{4 . 9 9}$ \\
& 0.2 & 1982 & 1096 & 3.34 & 1.84 \\
K6 & 2 & 1949 & 1053 & 59.2 & $\mathbf{3 2 . 6 2}$ \\
& 0.2 & 1996 & 873 & 2.7 & 1.48 \\
K7 & 2 & 1833 & 1043 & 53.4 & $\mathbf{2 9 . 4 2}$ \\
& 0.2 & 1889 & 938 & 2.78 & 1.53 \\
K8 & 2 & 1920 & 1045 & 73.3 & $\mathbf{4 0 . 3 9}$ \\
& 0.2 & 1804 & 853 & 6.14 & $\mathbf{3 . 3 8}$ \\
\hline Tariquidar & 2 & 1990 & 1131 & 65.5 & $\mathbf{3 6 . 0 9}$ \\
DMSO & 0.2 & 1957 & 1074 & 50.9 & $\mathbf{2 8 . 0 4}$ \\
\hline
\end{tabular}

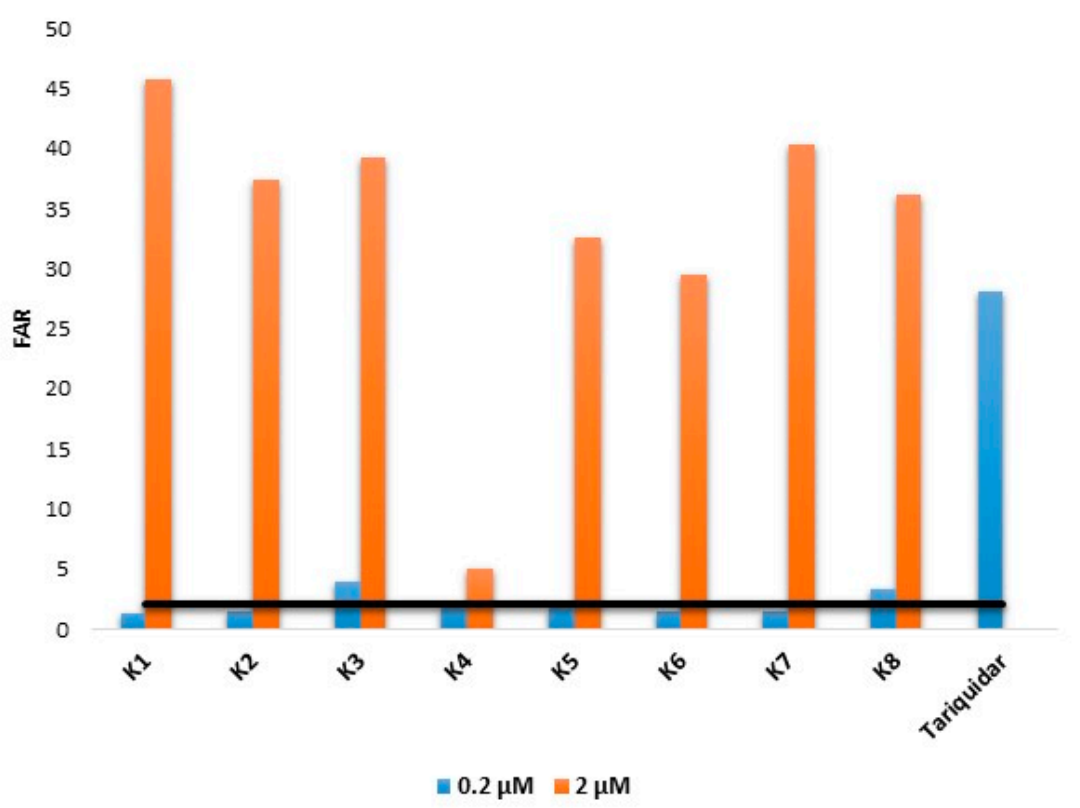

Figure 2. ABCB1 (P-gp) inhibition in the presence of selenoesters in MDR Colo 320 cells measuring the intracellular accumulation of the ABCB1 substrate rhodamine 123 by flow cytometry. The FAR (fluorescence activity ratio) values were calculated based on the equation given in Section 2.9. Tariquidar was applied as positive control; DMSO was used as solvent control. Results above FAR 2 (black line, highlighted in bold) are considered effective.

The parameters evaluated from flow cytometry experiments were: Forward Scatter Count (FSC, provides information about cell size); Side Scatter Count (SSC, proportional to cell granularity or internal complexity); FL-1 (Mean fluorescence of the cells), and Fluorescence Activity Ratio (FAR), which was calculated by the equation given above.

\subsection{P-gp ATPase Activity Assay}

Only the compounds with $\mathrm{ABCB} 1$ inhibitory activity were tested in this assay. As shown in Figure 3, $\triangle R L U$ of $\mathbf{K 1}, \mathbf{K} 7$, and $\mathbf{K} 8$ were significantly lower than $\triangle R L U_{\text {basal }}$, 
demonstrating that these compounds are inhibitors of P-gp ATPase activity. The rest of the compounds are stimulators of P-gp ATPase activity. Verapamil is a P-gp substrate that stimulates ATPase activity and served as a control in this assay. In the cases of $\mathbf{K} \mathbf{2}$ and $\mathbf{K} 6$, the P-gp ATPase activity could not be determined (Figure 3).

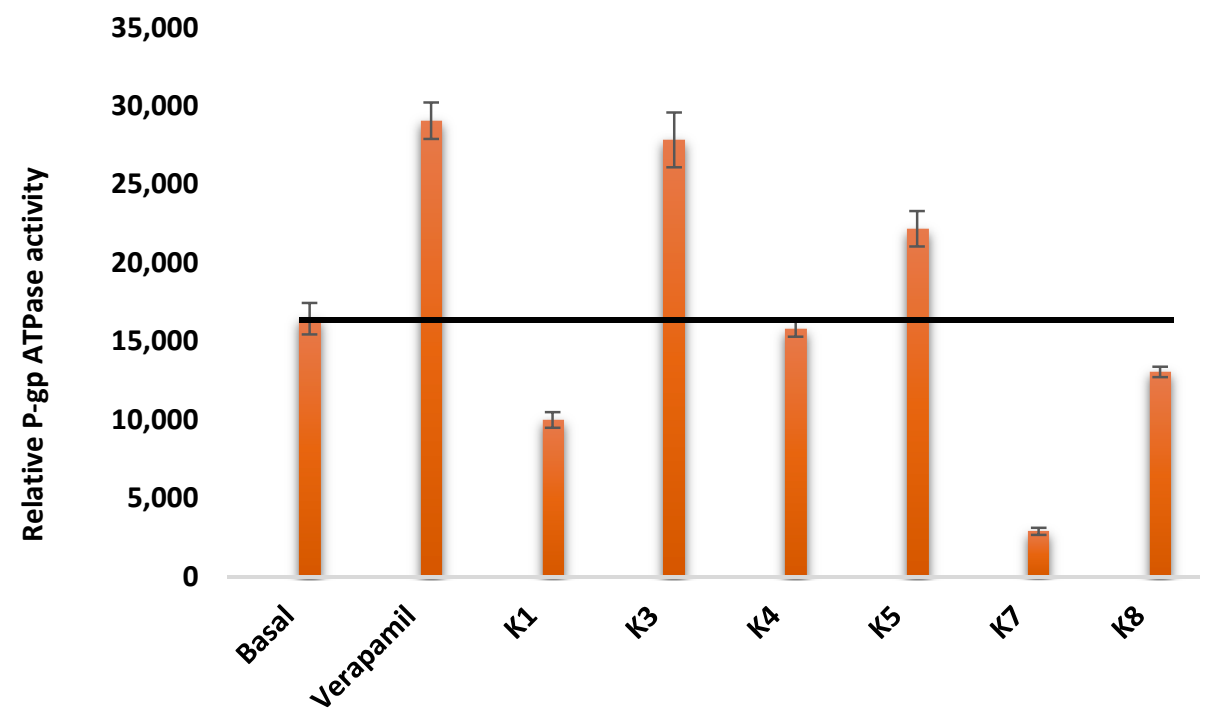

Figure 3. Relative ABCB1 (P-gp) ATPase inhibition activity of selected ketone-selenoesters. The effects are presented as the relative ATPase activity. The decrease in luminescence of untreated samples compared to samples treated with sodium-vanadate represents basal P-gp ATPase activity. The decrease in luminescence of verapamil-treated samples represents verapamil-stimulated P-gp ATPase activity. The lower the relative ATPase activity, the better the inhibitor. Results are calculated as the means \pm SD from experiments performed in triplicate. The level of basal activity is presented as a black line.

\subsection{Induction of Apoptosis}

The potent ABCB1 inhibiting ketone-selenoesters were tested regarding their apoptosisinducing activity on MDR Colo 320 colon adenocarcinoma cells (Table 7 and Figure 4).

The results were compared to $12 \mathrm{H}$-benzo $(\alpha)$ phenothiazine $(\mathrm{M} 627)$ as a positive control. The most potent compound, K3, could induce early apoptosis in $18.6 \%$ of the cell population, showing a higher capacity to trigger these early apoptotic events than the reference. Interestingly, all derivatives contributed to late apoptosis, and their activity ranged from 16.1 to $33.9 \%$. K3 induced late apoptosis in $25.6 \%$ of the cell population. Considering together the early and late apoptosis (as total apoptotic events), all of the compounds, with the exception of K4, induced apoptotic events in more than $30 \%$ of the gated cells. After defining the apoptosis quotient as the quotient of the total apoptotic events of the compound divided by the reference and expressed as a percentage, all of the compounds, except K4, showed an apoptosis induction ability equal to $64-90 \%$ more than the reference, and a concentration 10-fold lower than the reference. Compound $\mathbf{K} 3$ was the most potent with $89.5 \%$, followed by $\mathbf{K} 2$ with $69.2 \%$. The least effective was K4, with 39.5\%. During early apoptosis, phosphatidyl serine (PS) appears on the outer membrane, which can be detected by annexin V; however, the membrane has not yet disintegrated. During late apoptosis, the cell membrane is already damaged (in this case, annexin is also able to bind to PS), resulting in the release of DNA, to which propidium iodide is able to bind. 
Table 7. Apoptosis induction by ketone-selenoesters on MDR Colo 320 adenocarcinoma cells. The $12 H$ - benzo $(\alpha)$ phenothiazine (M627) was applied as a positive control.

\begin{tabular}{|c|c|c|c|c|c|c|}
\hline & $\begin{array}{l}\text { Conc. } \\
(\mu \mathrm{M})\end{array}$ & $\begin{array}{c}\text { Early Apoptosis (\%) } \\
\text { Treated } \\
\text { Sample-Untreated } \\
\text { Sample }\end{array}$ & $\begin{array}{c}\text { Late Apoptosis }(\%) \\
\text { Treated } \\
\text { Sample-Untreated } \\
\text { Sample }\end{array}$ & $\begin{array}{c}\text { Cell Death }(\%) \\
\text { Treated } \\
\text { Sample-Untreated } \\
\text { Sample }\end{array}$ & $\begin{array}{c}\text { Total Apoptotic } \\
\text { Events (Early + } \\
\text { Late, \%) }\end{array}$ & $\begin{array}{c}\text { Apoptosis } \\
\text { Quotient (\%) }\end{array}$ \\
\hline M627 & 20 & 13.3 & 36.1 & 0.00 & 49.4 & $100 \%$ \\
\hline K1 & 2 & 3.7 & 28.9 & 2.15 & 32.6 & $66.0 \%$ \\
\hline $\mathrm{K} 2$ & 2 & 0.3 & 33.9 & 0.95 & 34.2 & $69.2 \%$ \\
\hline K3 & 2 & 18.6 & 25.6 & 0.00 & 44.2 & $89.5 \%$ \\
\hline K4 & 2 & 3.4 & 16.1 & 5.95 & 19.5 & $39.5 \%$ \\
\hline K5 & 2 & 1.8 & 30.9 & 1.35 & 32.7 & $66.2 \%$ \\
\hline K6 & 2 & 4.5 & 27.8 & 0.17 & 32.3 & $65.4 \%$ \\
\hline K7 & 2 & 6.1 & 27.5 & 0.10 & 33.6 & $68.0 \%$ \\
\hline K8 & 2 & 4.9 & 26.7 & 0.85 & 31.6 & $64.0 \%$ \\
\hline
\end{tabular}

Apoptotic quotient is defined as the quotient of the total apoptotic events determined for the respective compound and the reference M627, expressed in percentage. The most effective early apoptosis induction is highlighted in bold.

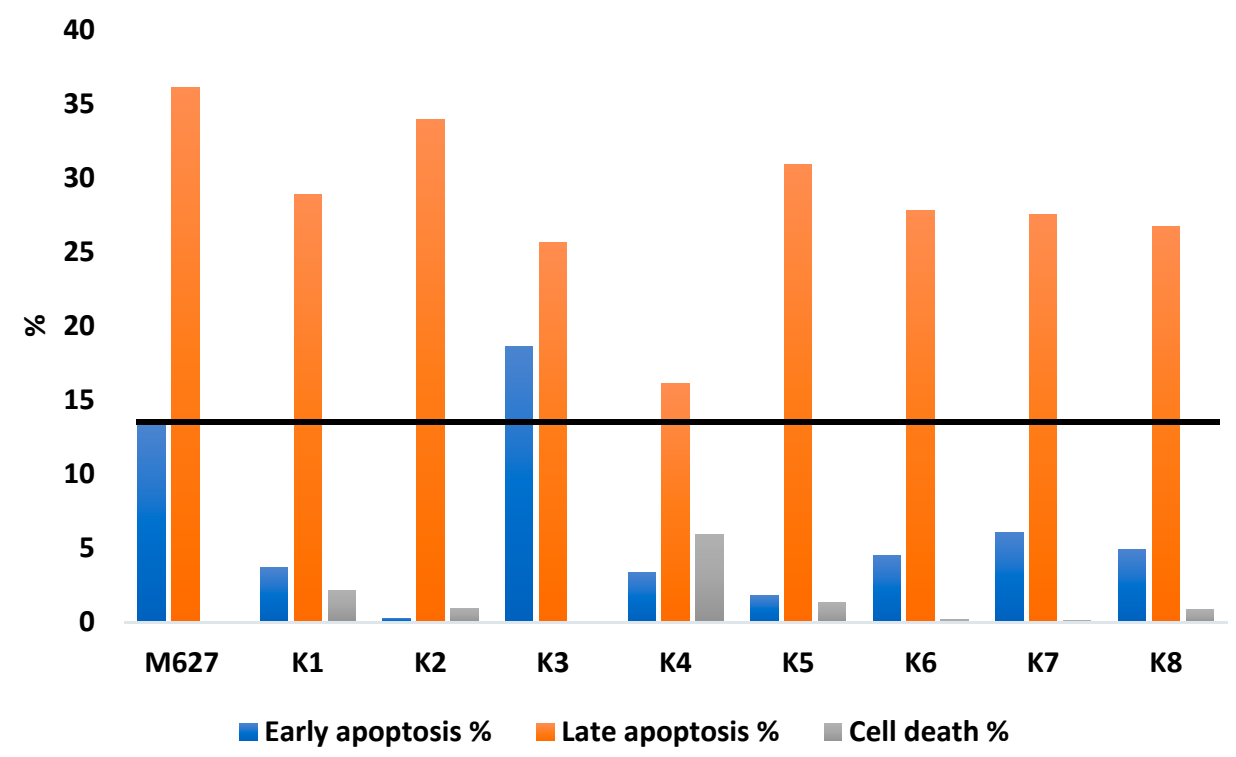

Figure 4. Apoptosis induction by ketone-selenoesters on MDR Colo 320 adenocarcinoma cells. The $12 \mathrm{H}$ - benzo $(\alpha)$ phenothiazine (M627) was applied as a positive control. The cell populations were analysed by flow cytometry after Annexin V-FITC and propidium iodine staining. The figure represents the percentage of early apoptotic cells (annexin positive, propidium iodide negative); late apoptotic cells (annexin positive, propidium iodide positive) ( $\mathrm{A}+, \mathrm{PI}+$ ); and dead cells (annexin negative, propidium iodide positive) (A-, PI+). The black line demonstrates the percentage of early apoptosis in the presence of the positive control M627.

\subsection{Wound Healing Assay}

Wound healing activity of selenoesters was evaluated as the ability of the cells to migrate and close the gap created on the monolayer of human keratinocytes (HaCaT cell line). The tested substances were applied in the highest possible non-toxic concentration ( $\mathrm{IC}_{10}$, concentration inhibiting $10 \%$ of the population). In general, ketone-selenoesters were more toxic (except K4 and K1) than cyano-selenoesters (except N4); therefore, they were applied at lower doses $(0.17-0.30 \mu \mathrm{M})$ when compared to cyanoselenoesters $(0.36-0.64 \mu \mathrm{M})$. Compounds K1, K4, and N4 were applied at a concentration of $0.30,0.46$, and $0.20 \mu \mathrm{M}$, respectively. As could be seen in Table 8, all ketone-selenoesters stimulated wound healing more effectively in comparison to both untreated control $(p<0.0000005)$ and allantoin $(p<0.00005)$. As a result, all of the tested ketone-selenoesters exhibited around $80 \%$ of gap closure within 24 h. After 48 h, samples K1, K2, K3, K5, K7, and K8 exhibited complete gap closure, and samples $\mathbf{K} 4$ and $\mathbf{K} 6$ were able to close the gap with a closure percentage 
of $86 \%$ and $93 \%$, respectively. In contrast, cyano-selenoesters were less active than ketoneselenoesters, as none of the samples were able to close the gap completely. Samples N3, N5, and N6 exhibited slower closure of the wound, and was slower than in the untreated control $(p<0.005)$ within $24 \mathrm{~h}$ (closure percentage was only around $45 \%)$. Only samples N1, N2, N4, and N7 closed effectively the damaged wound in $24 \mathrm{~h}$, with the gap closure around $70 \%$. Despite the fact that cyano-selenoesters were less potent in exhibiting the cell mobility for the wound healing, the samples N1, N4, and N7 were still even slightly better than the positive control $(p<0.005, p<0.05$, and $p<0.05$, respectively), and other cyano-selenoesters, with a closure percentage of around $80 \%$.

Table 8. Wound healing activity determined as keratinocytes' (HaCaT) wound closure (\%) after 24 and 48 h. Allantoin $(50 \mu \mathrm{g} / \mathrm{mL})$ served as a positive control. Data represent the average of twenty repetitions (four biological, five technical) with corresponding standard error of the mean. The data were analysed with $t$-test, where the difference between the group and negative control was considered statistically significant when $\left.p<0.0000005\left(^{* * * * *}\right), 0.000005{ }^{* * * * *}\right), 0.00005\left(^{* * * *}\right), 0.0005$ $\left({ }^{* * *}\right), 0.005(* *)$, and $0.05\left(^{*}\right)$.

\begin{tabular}{|c|c|c|c|c|c|c|c|}
\hline & Dose & & $24 \mathrm{~h}$ & & & $48 \mathrm{~h}$ & \\
\hline Compound & $\mathrm{IC}_{10}(\mu \mathrm{M})$ & Closure (\%) & SEM & $t$-Test & Closure (\%) & SEM & $t$-Test \\
\hline K1 & 0.30 & 83.64 & 1.03 & $* * * * * *$ & 100.00 & & $* * * * * * *$ \\
\hline $\mathrm{K} 2$ & 0.20 & 85.90 & 0.97 & $* * * * * *$ & 100.00 & & $* * * * * *$ \\
\hline K3 & 0.24 & 87.49 & 1.92 & $* * * * * *$ & 100.00 & & $* * * * * *$ \\
\hline K4 & 0.46 & 79.17 & 1.81 & $* * * * * *$ & 86.29 & 2.47 & $* * * * *$ \\
\hline K5 & 0.25 & 88.56 & 1.44 & $* * * * * *$ & 100.00 & & $* * * * * * *$ \\
\hline K6 & 0.22 & 83.02 & 1.54 & $* * * * * *$ & 92.65 & 1.34 & $* * * * * *$ \\
\hline K7 & 0.24 & 82.71 & 1.22 & $* * * * * *$ & 100.00 & & $* * * * * *$ \\
\hline K8 & 0.17 & 88.55 & 1.84 & $* * * * * *$ & 100.00 & & $* * * * * *$ \\
\hline N1 & 0.40 & 75.53 & 1.52 & $* * * * * *$ & 85.41 & 1.63 & $* * * * * *$ \\
\hline N2 & 0.36 & 72.23 & 1.20 & $* * * * * *$ & 77.51 & 1.98 & $*$ \\
\hline N3 & 0.64 & 46.46 & 2.00 & $* * * * * *$ & 75.70 & 1.44 & \\
\hline N4 & 0.20 & 73.65 & 1.92 & $* * * * * *$ & 83.84 & 2.09 & $* * * * * *$ \\
\hline N5 & 0.60 & 53.25 & 1.86 & $* *$ & 64.21 & 2.78 & $* * * * *$ \\
\hline N6 & 0.60 & 46.13 & 1.25 & $* * * * * *$ & 60.98 & 3.13 & $* * * * *$ \\
\hline N7 & 0.61 & 74.84 & 1.77 & $* * * * * *$ & 82.41 & 1.50 & $* * * * * *$ \\
\hline PC & & 70.37 & 2.56 & $* * * * * *$ & 84.63 & 2.21 & $* * * * * *$ \\
\hline NC & & 56.33 & 1.75 & & 75.55 & 1.71 & \\
\hline
\end{tabular}

SEM, standard error of the mean; $t$-test, 2-tailed $t$-test with unequal variances.

\section{Discussion}

\subsection{Synthesis and Characterization of the Compounds}

As mentioned in the Results section, the yield of the synthesis depends mostly on the purification procedure and on the matter state of the compound: generally, liquids are required to perform column chromatography, which reduces the yield. Solids showed a yield between $40 \%$ and $50 \%$, and liquids showed y yield between $7 \%$ and $29 \%$. Even for solids, the yield tends to be low. This may be related to the solubility of the compounds in water. As compounds are obtained (when they are solid) as a precipitate that is formed in the reaction media, part of the compound can remain in solution. Even knowing from previous works that selenoesters are poorly soluble in water, the usage of a significant volume during washings can be reflected in a significantly lower yield than the one expected.

The spectral data are fully described in the compound descriptions of Section 2.3, which discusses the chemical description of the compounds. Spectra enabled the correct assignments and the proper characterization of the structure of the compounds. As mentioned in the Results section, HMBC spectra were of particular interest to ensure the formation of the selenoester. A second proof was the isotopic pattern observed in the mass spectra of the oxoselenoesters in which the molecular ion has a higher abundance, as indicated by $\mathbf{K} 2, \mathbf{K} 5, \mathbf{K} 6$, and $\mathbf{K 8}$. Another indirect proof is the observation in ${ }^{13} \mathrm{C}-\mathrm{NMR}$ 
that the range of the peak chemical shift is always narrower for the carbonyl of the ketone or for the cyano group than for the carbonyl of the selenoesters. This is logical as the ketone or the cyano group are at a higher distance from the ring substituents, which are more responsible for the observed variations, than the carbonyl of the selenoester. A similar observation was found with the range of the bond stretches, $\mathrm{C}=\mathrm{O}\left(\mathrm{COCH}_{3}\right)$ and $\mathrm{C} \equiv \mathrm{N}$, as compared to the $\mathrm{C}=\mathrm{O}(\mathrm{COSe})$ stretch.

\subsection{Anticancer Activity}

Oxoselenoesters, in general (a few exceptions are observed), showed a more potent cytotoxicity than cyanoselenoesters in MDR Colo 320, HepG2, and B16 cancer cell lines. This difference of activity is particularly marked in Colo 320 and HepG2 cells. Cyanoselenoesters showed no activity on the MRC-5 cells; however, they were very toxic on both colon cancer cell lines, and were less potent on the resistant cells. Regarding the most active compound in each cell line (the second and third most active in brackets after cell line), these were as follows: K4 in Colo 205 (K1, N4); K1 in Colo 320 (K4, K6); K2 in HepG2 (K1, K5); N6 in HeLa (K4, K3); and K5 in B16 cells (K2, K6). In line with the overall impression, the majority of them are oxoselenoesters, and among them, all appeared at least once, except K7 and K8. This may point out that the presence of substituents without halogen(s) - as is the case of K7-reduces the activity, as the compounds that include a trifluoromethyl group or that have one or two halogens bound to the phenyl ring showed better activity. However, the inclusion of a third fluorine at the phenyl ring (K8) is also less profitable for the cytotoxicity. On the other hand, the recurrent appearance of $\mathbf{K} \mathbf{1}$ and K4 among the most active compounds (three times each) against each cell line points out that the thionyl ring favours the cytotoxicity, as well as the presence of a trifluoromethyl group in ortho position (in the phenyl ring) in respect to the selenoester. Interestingly, K5 (which has a $3-\mathrm{CF}_{3}$ substituent at the ring) also exerts noteworthy activity, and the cyanoselenoester, N4, with a $2-\mathrm{CF}_{3}$ substituent at the ring, is perhaps the most active derivative among the cyanoselenoesters. This supports the empirical observation of the relevance of this trifluoromethyl group (preferable in ortho position, although the compound with this substituent in meta position has a comparable activity in all cell lines, except in HepG2, in which it is significantly less active) for the cytotoxic activity.

The ketone-containing selenoesters showed toxicity towards normal MRC-5 cells, whereas none of the cyano-containing derivatives exerted toxicity against this cell line at concentrations below $100 \mu \mathrm{M}$. This implies that all of the cyanoselenoesters were strongly selective $(\mathrm{SI}>6)$ towards cancer cells. They were especially selective towards B16 skin melanoma cells, as N4 and N5 showed an SI higher than 71.4, and N7 showed an SI higher than 62.5. Compound N6 was also extremely selective towards HeLa cervical adenocarcinoma cells (SI >76.9). More compounds showed SI values higher than 50 towards cancer cells: N1 and N6 in B16 cells, N1 in HeLa cells, and N4 and N7 in Colo 205 cells. Ketone-selenoesters, in contrast, were much less selective. Only K4 showed a moderate selectivity towards Colo205 cells $(3<$ SI $<6)$. The remaining compounds were slightly selective $(1<\mathrm{SI}<3)$ towards the tested cancers cells in respect to MRC-5 non-cancer cells. Even a few ones were non-selective (SI < 1): K3, K4, and K6 towards HepG2 cells, and $\mathrm{K} 2$ towards Colo205 cells. This indicates a risk of side effects, and further research is necessary to find compounds with a similar potency and more selectivity.

Regarding the combination of ketone-selenoesters and cyanoselenoesters with the cytotoxic drug doxorubicin, eleven of the fifteen selenoesters evaluated interacted in a synergistic manner with doxorubicin in at least one of the ratios tested: K1, K3, K4, K5, K6, K8, N1, N2, N3, N4, and N7. No logical structure-activity relationships (SARs) can be extracted, as $\mathbf{K} \mathbf{5}$ and $\mathbf{K} 6$ interacted in different synergism degrees with doxorubicin in all of the ratios, whereas their nitrile equivalents (N5 and N6) showed antagonistic interactions for five of the six ratios tested. Similarly, the thiophene cyanoselenoester N1 showed a synergistic interaction with doxorubicin in four of the six ratios assayed, while its ketone 
analogue $\mathbf{K} \mathbf{1}$ only showed synergism in one ratio out of five. This $\mathbf{N} 1$ derivative was also capable of interacting with a strong synergism, at a 54.4:1 ratio with doxorubicin.

To reverse efflux-related MDR, the inhibition of the ABCB1 pump was investigated in the presence of selenocompounds. The 2-oxopropyl moiety of the ketone-selenoesters is crucial for the activity, as all ketone-selenoesters, except K4, were more potent ABCB1 inhibitors, whereas none of the cyanoselenoesters showed ABCB1 inhibiting activity. This is also in line with previous works $[15,16]$, in which both this moiety and a 3,3-dimethyl-2oxobutyl moiety showed very potent activity.

The most active compound is $\mathbf{K} \mathbf{1}$, which contains a thiophene ring instead of a phenyl ring. In this case, the insertion of a bulky trifluoromethyl group at the two-position of the phenyl ring significantly reduced the activity (K4): simply moving this group to the threeposition and eliminating the steric hindrance produced a sixfold increase of the activity (K5). Interestingly, two oxoselenoesters that showed a less marked cytotoxicity (K7, with a 4-tert-butylphenyl ring; and K8, with a 2,4,5-trifluorophenyl ring) were strong inhibitors of $\mathrm{ABCB} 1$, with a similar activity to $\mathbf{K} \mathbf{2}$ and $\mathbf{K} 3$, with 2-fluorophenyl and 4-bromophenyl moieties, respectively.

Regarding the P-gp ATPase activity, only the oxoselenoesters were tested as they were the only ones with ABCB1 inhibitory activity. Among them, the P-gp ATPase activity of K2 and $\mathbf{K} 6$ could not be determined, and all of the remaining tested compounds, except K4 (whose activity was similar to the one observed for the basal control), modulated the ATPase activity. Interestingly, $\mathbf{K} \mathbf{1}$ and $\mathbf{K} 7$, which where the most potent ABCB1 inhibitors, inhibited the ATPase activity, especially in the case of K7. Besides, K8 exerted a milder inhibition of the ATPase. Since the activity of ABCB1 can protect the cells from apoptosis, the inhibition of the energy supply of this pump can promote apoptosis, as demonstrated by our results [21]. The abovementioned derivatives (K1, K7, and K8) induced late apoptosis in MDR Colo 320 cells, confirming the connection between ABCB1 inhibition and apoptosis induction.

On the other hand, K3 stimulated the P-gp ATPase with a comparable intensity to the reference verapamil, whereas $\mathbf{K} 5$ produced a milder stimulation. Then-keeping in mind that the data available are scarce-with this data, the thienyl ring and the 4-tertbutylphenyl and 2,4,5-trifluorophenyl moieties inhibited the ATPase activity, whereas the 4-bromophenyl and the 3-(trifluoromethyl)phenyl moiety stimulated it. Finally, moving the trifluoromethyl group from the three- to the two-position eliminated this promotion of the ATPase activity.

The connection between ABCB1 inhibition and apoptosis induction was investigated in the case of ketone-selenoesters. The ketone-selenoesters showed a significant ability to trigger apoptotic events, with the exception of $\mathbf{K} 4$. Compound $\mathrm{K} 3$ was more effective than the reference phenothiazine in the induction of early apoptosis, and is the one that, considering together the early and late apoptosis, induced apoptosis with a potency closer to the reference $(89.5 \%)$. As all of the remaining derivatives assayed showed a similar ability to induce apoptosis, no SARs can be extracted with the available data. Besides, it is evident that the inclusion of a bromine atom at the four-position of the phenyl ring increases the apoptosis induction (K3), and the inclusion of a bulky substituent at the two-position (as the trifluoromethyl moiety) of the phenyl ring reduces the ability to trigger apoptotic events. In addition, the presence of selenium can induce the formation of free radicals, resulting in apoptosis and cell death in cancer cells [22-24]. The functions of MDR transporter proteins (most notably ABCB1) have been described in apoptosis evasion, mediated by a dampening of the extrinsic apoptotic pathway (through suppression of TRAIL protein and caspases three and eight) and the stabilization of cell membrane phospholipids (through acting as an outwardly directed flippase). The inter-relatedness of overexpressed efflux pumps and programmed cell death may explain the results obtained in the apoptosis detection assay [21].

In general, selenium in the form of selenoproteins and selenocompounds has always been known as an excellent candidate for wound healing, as some of them have exhibited 
their ability to act as antioxidants and inhibitors of inflammation. Selenocompounds act as inhibitors of cytokines and eliminators of peroxynitrate, which is a super radical ion in the inflammatory phase [25]. As described in the results, all of the eight ketoneselenoesters stimulated an effective wound healing process, demonstrating a better healing ability than the positive control allantoin. Six of them (K1, K2, K3, K5, K7, and K8) were even capable of repairing the wound completely. Only the compounds with a bulky substituent in the ortho position (trifluoromethyl, K4) or with two different substituents (K6, 3-chloro-4-fluorophenyl) were not capable of completely closing the wound. Regarding the cyano-selenoesters, they were significantly less effective in this assay than the ketoneselenoesters. Only one of the seven cyano-containing derivatives, compound N1, displayed better closure than allantoin (especially after 24). Three additional ones, N2, N4, and N7, were more effective than the negative control. In this case, no reliable SARs can be extracted, but the removal of one of the two trifluoromethyl substituents from compound N7 resulted in significant wound healing activity loss, which serves as the main reason for the inability of compound N5 to close the gap. Similarly, compound N6 and N3 had the same effect as compound N5.

If a few compounds would need to be selected among all derivatives to proceed to more in-depth studies, perhaps the most promising are $\mathbf{K} \mathbf{1}, \mathbf{K} \mathbf{3}$, and $\mathbf{K} \mathbf{5}$. The oxoselenoester $\mathbf{K 1}$, which has a thiophene ring bound to the carbonyl of the selenoester, is a potent cytotoxic compound that exerts a strong inhibition of the ABCB1 efflux pump and of the ATPase activity, and also has the ability of apoptosis induction and the capacity to promote a complete closure of a wound. Similarly, the 2-oxopropyl 4-bromobenzoselenoate (K3) has a noteworthy cytotoxic activity, and strongly inhibits the ABCB1 efflux pump, but stimulates the ATPase activity. Besides, it is the most potent apoptosis inducer among the tested compounds and also manages to complete the closure of a wound in $48 \mathrm{~h}$. Finally, the 2-oxopropyl 3-(trifluoromethyl)benzoselenoate (K5) has a similar effect than K3, but with a less potent apoptosis-inducing ability and less capacity to enhance P-gp ATPase activity. In exchange, it could interact in a synergistic manner with doxorubicin when administered in combination, in the six ratios tested. Between these three derivatives, all of the activities tested are covered, as are the two ways of action in the case of the ATPase assay. The compound 2-oxopropyl 3-(trifluoromethyl)benzoselenoate (K4) seemed to be a promising derivative in cytotoxicity assay, but later showed a poor effectivity in ABCB1 inhibition, ATPase modulation, apoptosis induction, and wound healing, so it is clearly a less multitarget compound than K1, K3, and K5. Perhaps the presence of a bulky substituent in a position close to the selenoester can affect the interaction of the selenium atom with the different cellular targets. On the other hand, it may affect its hydrolysis, as this is the hypothesized mechanism of action for these compounds, according to previous works [16].

In contrast, the cyanoselenoesters are more selective compounds, but they are generally less cytotoxic, weaker promoters of wound healing, interacted in a less synergistic manner with doxorubicin in combination assay, and did not inhibit the ABCB1 protein.

\section{Conclusions}

Herein, we described the design, synthesis, and characterization of fifteen novel selenoesters, as well as the evaluation of their activity against a wide selection of different targets related to cancer multidrug resistance. Of these selenoesters, the alkyl moiety of eight included a ketone group, whereas the seven remaining contained a cyano group. All of the compounds showed $\mathrm{IC}_{50}$ values between 1 and $12 \mu \mathrm{M}$ in the five cancer cell lines evaluated. The oxoselenoesters were generally more cytotoxic, while the cyanoselenoesters were more selective towards cancer cells in respect to non-cancer cells. Besides, the majority of the obtained oxoselenoesters were potent ABCB1 inhibitors, enhanced the activity of doxorubicin in a synergistic manner (at least in any of the ratio concentrations tested), and modulated the P-gp ATPase activity. All of the oxoselenoesters showed an apoptosis induction capacity and an ability to promote wound healing. Therefore, these 
novel selenocompounds have shown noteworthy multi-target anticancer activity that converts them into a promising starting point to develop more effective and selective anticancer agents.

Supplementary Materials: The following are available online at https:/ / www.mdpi.com/article/10 .3390/cancers13184563/s1. Figure S1: Compound K1: Se-(2-oxopropyl) thiophene-2-carboselenoate, Figure S2: Compound K2: Se-(2-oxopropyl) 2-fluorobenzoselenoate, Figure S3: Compound K3: Se-(2oxopropyl) 4-bromobenzoselenoate, Figure S4: Compound K4: Se-(2-oxopropyl) 2-(trifluoromethyl) benzoselenoate, Figure S5: Compound K5: Se-(2-oxopropyl) 3-(trifluoromethyl)benzoselenoate, Figure S6: Compound K6: Se-(2-oxopropyl) 3-chloro-4-fluorobenzoselenoate, Figure S7: Compound K7: Se-(2-oxopropyl) 4-(tert-butyl)benzoselenoate, Figure S8: Compound K8: Se-(2-oxopropyl) 2,4,5trifluorobenzoselenoate, Figure S9: Compound N1: Se-(cyanomethyl) thiophene-2-carboselenoate, Figure S10: Compound N2: Se-(cyanomethyl) 3-fluorobenzoselenoate, Figure S11: Compound N3: Se-(cyanomethyl) 4-bromobenzoselenoate, Figure S12: Compound N4: Se-(cyanomethyl) 2(trifluoromethyl)benzoselenoate, Figure S13. Compound N5: Se-(cyanomethyl) 3-(trifluoromethyl) benzoselenoate, Figure S14. Compound N6: Se-(cyanomethyl) 3-chloro-4-fluorobenzoselenoate, Figure S15. Compound N7: Se-(cyanomethyl) 3,5-bis(trifluoromethyl)benzoselenoate.

Author Contributions: Conceptualization, G.S., J.V., and E.D.-Á.; methodology, J.V., G.S., and E.D.-Á.; chemical synthesis and analysis, N.S.-J., C.S.-H., M.B.-L., F.-J.A.-M., and E.D.-Á; biological evaluation and data processing, N.S., S.D., G.H., A.K., and M.N.; writing-original draft preparation, N.S.-J., S.D., G.S., J.V., and E.D.-Á.; writing—review and editing, G.S., J.V., and E.D.-Á.; supervision, G.S., J.V., and E.D.-Á.; project administration, J.V., G.S., and E.D.-Á.; funding acquisition, G.S., J.V., and E.D.-Á. All authors have read and agreed to the published version of the manuscript.

Funding: The study was supported by the projects SZTE ÁOK-KKA 2018/270-62-2 of the University of Szeged, Faculty of Medicine and GINOP-2.3.2-15-2016-00038 (Hungary); and Consejo Superior de Investigaciones Científicas (CSIC, Spain, project LINKA20285). This research was funded by VISEGRAD FUND, grant number 22010090; and by the mobility project from the Czech Ministry of Education, Youth and Sports INTER-COST, grant number LTC19007. This article is based upon work from COST Action $17104<$ STRATAGEM>, supported by COST (European Cooperation in Science and Technology), (http:/ / www.cost.eu, accessed on 17 September 2021). The study was supported also by two cultural associations: "Trevinca" and "Iniciativas Ropelanas".

Institutional Review Board Statement: Not applicable.

Informed Consent Statement: Not applicable.

Data Availability Statement: The data presented in this study are available in this article (and supplementary material). Additional data are available on request from the corresponding author.

Conflicts of Interest: The authors declare no conflict of interest. The funders had no role in the design of the study; in the collection, analyses, or interpretation of data; in the writing of the manuscript; or in the decision to publish the results.

\section{References}

1. Bellamy, W.T. P-Glycoproteins and multidrug resistance. Annu. Rev. Pharmacol. Toxicol. 1996, 36, 161-183. [CrossRef] [PubMed]

2. Smyth, M.J.; Krasovskis, E.; Sutton, V.R.; Johnstone, R.W. The drug efflux protein, P-Glycoprotein, additionally protects drugresistant tumor cells from multiple forms of caspase-dependent apoptosis. Proc. Natl. Acad. Sci. USA 1998, 95, 7024-7029. [CrossRef]

3. Sharom, F.J.; Liu, R.; Romsicki, Y.; Lu, P. Insights into the structure and substrate interactions of the P-Glycoprotein multidrug transporter from spectroscopic studies. Biochim. Biophys. Acta (BBA)—Biomembr. 1999, 1461, 327-345. [CrossRef]

4. Brinkmann, U. Functional Polymorphisms of the Human Multidrug Resistance (MDR1) Gene: Correlation with P Glycoprotein Expression and Activity in vivo. In Novartis Foundation Symposia; Bock, G., Goode, J.A., Eds.; John Wiley \& Sons, Ltd.: Chichester, UK, 2008; pp. 207-212. ISBN 978-0-470-84146-4.

5. Pokharel, D.; Roseblade, A.; Oenarto, V.; Lu, J.F.; Bebawy, M. Proteins regulating the intercellular transfer and function of P-Glycoprotein in multidrug-resistant cancer. Ecancer 2017, 11, 768. [CrossRef] [PubMed]

6. Hiller, D.; Sanglard, D.; Morschhäuser, J. Overexpression of the MDR1 Gene is sufficient to Confer increased resistance to toxic compounds in Candida Albicans. Antimicrob. Agents Chemother. 2006, 50, 1365-1371. [CrossRef] [PubMed]

7. Barrand, M.A.; Bagrij, T.; Neo, S.-Y. Multidrug resistance-associated protein: A protein distinct from P-Glycoprotein involved in cytotoxic drug expulsion. Gen. Pharmacol. Vasc. Syst. 1997, 28, 639-645. [CrossRef] 
8. Allen, J.D.; Brinkhuis, R.F.; van Deemter, L.; Wijnholds, J.; Schinkel, A.H. Extensive contribution of the multidrug transporters P-Glycoprotein and Mrp1 to basal drug resistance. Cancer Res. 2000, 60, 5761-5766.

9. Allen, J.D.; Van Dort, S.C.; Buitelaar, M.; van Tellingen, O.; Schinkel, A.H. Mouse breast cancer resistance protein (Bcrp1/Abcg2) mediates etoposide resistance and transport, but etoposide oral availability is limited primarily by P-Glycoprotein. Cancer Res. 2003, 63, 1339-1344.

10. Palmeira, A.; Sousa, E.; Vasconcelos, M.H.; Pinto, M.; Fernandes, M.X. Structure and ligand-based design of P-Glycoprotein inhibitors: A historical perspective. Curr. Pharm. Des. 2012, 18, 4197-4214. [CrossRef] [PubMed]

11. Prabhu, K.S.; Lei, X.G. Selenium. Adv. Nutr. 2016, 7, 415-417. [CrossRef]

12. Vinceti, M.; Filippini, T.; Cilloni, S.; Crespi, C.M. The Epidemiology of Selenium and Human Cancer. In Advances in Cancer Research; Elsevier: Cambridge, MA, USA, 2017; Volume 136, pp. 1-48. ISBN 978-0-12-812016-3.

13. Álvarez-Pérez, M.; Ali, W.; Marć, M.A.; Handzlik, J.; Domínguez-Álvarez, E. Selenides and diselenides: A review of their anticancer and chemopreventive activity. Molecules 2018, 23, 628. [CrossRef]

14. Maiyo, F.; Singh, M. Selenium nanoparticles: Potential in cancer gene and drug delivery. Nanomedicine 2017, 12, 1075-1089. [CrossRef] [PubMed]

15. Domínguez-Álvarez, E.; Gajdács, M.; Spengler, G.; Palop, J.A.; Marć, M.A.; Kieć-Kononowicz, K.; Amaral, L.; Molnár, J.; Jacob, C.; Handzlik, J.; et al. Identification of selenocompounds with promising properties to reverse cancer multidrug resistance. Bioorganic Med. Chem. Lett. 2016, 26, 2821-2824. [CrossRef] [PubMed]

16. Gajdács, M.; Spengler, G.; Sanmartín, C.; Marć, M.A.; Handzlik, J.; Domínguez-Álvarez, E. Selenoesters and selenoanhydrides as novel multidrug resistance reversing agents: A confirmation study in a colon cancer MDR cell line. Bioorganic Med. Chem. Lett. 2017, 27, 797-802. [CrossRef] [PubMed]

17. Spengler, G.; Gajdács, M.; Marć, M.; Domínguez-Álvarez, E.; Sanmartín, C. Organoselenium compounds as novel adjuvants of chemotherapy drugs-A promising approach to fight cancer drug resistance. Molecules 2019, 24, 336. [CrossRef]

18. Gajdács, M.; Nové, M.; Csonka, Á.; Varga, B.; Sanmartín, C.; Domínguez-Álvarez, E.; Spengler, G. Phenothiazines and selenocompounds: A potential novel combination therapy of multidrug resistant cancer. Anticancer Res. 2020, 40, 4921-4928. [CrossRef]

19. Liang, C.-C.; Park, A.Y.; Guan, J.-L. In vitro scratch assay: A convenient and inexpensive method for analysis of cell migration in vitro. Nat. Protoc. 2007, 2, 329-333. [CrossRef]

20. Chou, T.-C. Drug combination studies and their synergy quantification using the Chou-Talalay method. Cancer Res. 2010, 70, 440-446. [CrossRef]

21. Stavrovskaya, A.A.; Moiseeva, N.I. Non-canonical functions of the cellular transporter P-Glycoprotein. Biochem. Mosc. Suppl. Ser. A 2016, 10, 241-250. [CrossRef]

22. Zhu, Z.; Kimura, M.; Itokawa, Y.; Aoki, T.; Takahashi, J.A.; Nakatsu, S.; Oda, Y.; Kikuchi, H. Apoptosis induced by selenium in human glioma cell lines. Biol. Trace Element Res. 1996, 54, 123-134. [CrossRef]

23. Ghosh, J. Rapid induction of apoptosis in prostate cancer cells by selenium: Reversal by metabolites of arachidonate 5lipoxygenase. Biochem. Biophys. Res. Commun. 2004, 315, 624-635. [CrossRef] [PubMed]

24. Zu, Y.; Yang, Z.; Tang, S.; Han, Y.; Ma, J. Effects of P-Glycoprotein and its inhibitors on apoptosis in K562 cells. Molecules 2014, 19, 13061-13075. [CrossRef] [PubMed]

25. Hariharan, S.; Dharmaraj, S. Selenium and selenoproteins: It's role in regulation of inflammation. Inflammopharmacology 2020, 28, 667-695. [CrossRef] [PubMed] 